ARCHIV FÜR MUSIKWISSENSCHAFT 76, 2019/2, 135-162

DOI $10.25162 /$ AFMW-2019-0007

MARK DELAERE

\title{
Eine angewandte serielle und elektronische Musik?
}

Stockhausen, Goeyvaerts und die musikalische Liturgiereform um 1955

\author{
A Functional Use of Electronic and Serial Music? \\ Stockhausen, Goeyvaerts, and the Music-Liturgical Reform of 1955
}

\begin{abstract}
It is well known that Karlheinz Stockhausen and Karel Goeyvaerts were imbued with the Catholic faith. For both composers, the development of serial music primarily arose from musical considerations, but beyond that, the invention of new compositional techniques also constituted an act of faith. Although their relationship to religion was at first essentially a private affair, limited to idealistic conceptions of music as representation of the Divine, both composers began to develop an interest in the functional use of serial and electronic music in the Catholic liturgy around 1955. The present article reconstructs this turn on the basis of recently published mutual correspondence and other archival materials. Included are also analyses of planned (Stockhausen) and realized (Goeyvaerts) mass compositions.

Keywords: Gesang der Jünglinge, Mis ter nagedachtenis van Zijne Heiligheid Paus Johannes XXIII, Catholic liturgy, compositions based on the mass
\end{abstract}

Am 22. Dezember 1956 schreibt Karel Goeyvaerts an Karlheinz Stockhausen:

Seit einiger Zeit korrespondiere ich mit einem Benediktinermönch, Dom A[mbroos] Verheul, dem Leiter der flämischen liturgischen Zeitschrift. Im Frühjahr wird in seinem Kloster ein der liturgischen Musik gewidmeter Studientag belegt [veranstaltet]. Er ist ja sehr begeistert von meinen Vorschlägen; ich fürchte aber, dass er sich noch nicht ganz gut [genau] vorstellt, worum es sich handelt. Karlheinz, würde es Dich interessieren, unseren Studientag mitzumachen? ${ }^{1}$

Wie bekannt, waren beide Komponisten von dem katholischen Glauben geprägt. Sicher, die Entwicklung der seriellen Musik erschien für sie vor allem aus musikalischen Gründen unausweichlich, aber darüber hinaus stellte die Erfindung neuer Kompositionstechniken für sie auch einen Glaubensakt dar. Die Beziehung zur Religion war für sie anfänglich Privatsache und auf idealistische Vorstellungen von Musik als Darstellung des Göttlichen beschränkt. Um 1955 interessierten sich aber sowohl Stockhausen wie Goeyvaerts für eine mögliche funktionale Anwendung der neuen Musik in der

1 Imke Misch, Mark Delaere (Hg.), Karel Goeyvaerts - Karlheinz Stockhausen. Briefwechsel/Correspondence 1951-1958, Kürten 2017, S. 203. 
katholischen Liturgie. Vorliegender Beitrag rekonstruiert diese Wendung zu einer angewandten seriellen und elektronischen Musik anhand der jüngst erschienenen Korrespondenz zwischen den beiden Komponisten und weiterer Archivunterlagen. Auch die Ergebnisse dieser Wendung in Form von geplanten, beziehungsweise ausgeführten kirchenmusikalischen Kompositionen von Stockhausen und Goeyvaerts werden untersucht ${ }^{2}$.

\section{Biografischer Kontext}

Karel Goeyvaerts (1923-1993) entstammt einer Antwerpener Familie, in der der Katholizismus fest verwurzelt war. Sein ältester Bruder war Priester, und auch der zwölffährige Karel wurde zuerst in das „Klein Seminarie“ in Mecheln aufgenommen. Sobald er aber erfuhr, dass seine neue Schule explizit auf eine Priesterausbildung vorbereitete, versuchte er dort wegzukommen, und bereits 1936 gelang es ihm, auf eine andere, zwar immer noch katholische, aber nicht auf das Priesteramt vorbereitende Schule zu wechseln. Nach dem Gymnasium wollte er sich als Komponist ausbilden, wurde aber zuerst in das „Lemmens-Instituut“ in Mecheln immatrikuliert, damals eine fast ausschließlich auf die Ausbildung von Kirchenmusikern im Sog des Cäcilianismus ausgerichtete Musikhochschule. Weder hier noch in dem „Koninklijk Conservatorium Antwerpen“, auf das er bald wechselte, fand Goeyvaerts aber die ersehnte Ausbildung in der neuen Musik, sodass er ab Oktober 1947 im Pariser „Conservatoire National“ Komposition bei Darius Milhaud und Analyse bei Olivier Messiaen studierte. Messiaen gilt natürlich wie kein anderer als ein Komponist, der während seiner gesamten Laufbahn, von Le banquet céleste (1928) bis zu Saint François d'Assise (1975-1983) und Éclairs sur l'Au-Delà ... (19871991), versuchte, ein katholisches Programm mit neuer Musik zu verknüpfen ${ }^{3}$. Es ist bezeichnend, dass Goeyvaerts nicht darum bemüht war, seinen katholischen Hintergrund mit Messiaens ästhetischer Theologie in Verbindung zu bringen. Stattdessen betonte er ausschließlich die neue musikalische Denkweise von Messiaen, wie sie sich aus seinen Analysen von Werken wie etwa Strawinskys Le sacre du printemps oder aus seinen Kompositionen wie Mode de valeurs et d'intensités ablesen ließ. Es handelt sich dabei um die

2 Camilla Bork, Diederik Verstraete, Klaas Coulembier, Els T'Seyen, Jan Christiaens, Imke Misch, Maria Luckas und Kathinka Pasveer der Stockhausen Stiftung für Musik seien für ihre Hilfe bei der Genese dieses Beitrags herzlichst gedankt.

3 Die Literatur über Messiaens klingende Theologie ist inzwischen fast unübersichtlich und lässt nicht nach. Verwiesen sei hier lediglich auf Almut Rößler, Beiträge zur geistigen Welt Olivier Messiaens, Duisburg 1984; Clytus Gottwald, Neue Musik als spekulative Theologie. Religion und Avantgarde im 2o. Jahrhundert, Stuttgart 2003; Siglind Bruhn, Messiaens Summa theologica: Musikalische Spurensuche mit Thomas von Aquin in La transfiguration, Méditations und Saint François d'Assise, Waldkirch 2008; Michaela Christine Hastetter (Hg.), Musik des Unsichtbaren. Der Komponist Olivier Messiaen (1908-1992) am Schnittpunkt von Theologie und Musik, St. Ottilien 2008; Siglind Bruhn, Messiaen's Interpretations of Holiness and Trinity: Echoes of Medieval Theology in the Oratorio, Organ Meditations, and Opera, Hillsdale 2008; Sander van Maas, The Reinvention of Religious Music: Olivier Messiaen's Breakthrough Toward the Beyond, New York 2009; Andrew Shenton (Hg.), Messiaen the Theologian, London 2010; Thomas Schrader, „Was kein Ohr gehört hat": Eine Untersuchung der Musik Messiaens aus musikwissenschaftlicher und theologischer Sicht, Augsburg 2011. 
objektive Betrachtung sämtlicher Tonparameter und deren präkompositorische Vorordnung. Obschon die übergroße Mehrzahl von Messiaens Werken auf Klangschönheit und Ausdrucksgestik zielt, sei diese Produktion laut Goeyvaerts eher sekundär:

Der wirkliche Messiaen, wie er sich in seinen letzten Werken zeigt, wie er auch in seiner Lehre hervortritt, der Messiaen, den wir erwarten können, ist also in die Evolution unserer Zeit eingeschaltet, in die Entwicklung, die sich von dem Individuellen in das Kollektive, von der aktiven in die passive Geisteshaltung, von dynamischen in statische Strukturen verwandelt hat ${ }^{4}$.

Goeyvaerts hat seine Vorstellungen von dem „wirklichen Messiaen“ dann mit seiner Interpretation eines „wirklichen Anton Webern“ produktiv verbunden, um mit seinem zweiten Violinkonzert (1951) und Nr. 1 (Sonate für 2 Klaviere) (Winter 1950-1951) noch annähernd, mit seiner Nr. 2 für 13 Instrumente (August-September 1951) dann aber vollends die ersten Beispiele des europäischen integralen oder multiparametrischen Serialismus zu kreieren. Goeyvaerts' serielle und elektronische Arbeiten aus den Jahren 1950 stellen einen Versuch dar, sich von der dynamischen, Ich-bezogenen Ausdrucksmusik abzuwenden und einer statischen Musik als Abbild des Göttlichen näherzukommen. In seiner ab 1959 anzusetzenden postseriellen Produktion probiert Goeyvaerts nicht länger, das Sakrale durch eine kompromisslose, „reine“ Musiksprache darzustellen, sondern verarbeitet explizit christliche Thematiken auf musikalisch verschiedene, durchaus aber auch weniger strikt durchorganisierte Weise. Auffallend dabei ist seine Neigung zu den „sadomasochistischen“ Aspekten des Katholizismus. Fast all seine explizit religiös inspirierten Kompositionen befassen sich tatsächlich mit der Folterung, dem Leiden und dem Tod Christi: Improperia. Kantate zum Karfreitag (1959) als erstes postserielles Werk, das Orchesterwerk Die Passion (1962) und Ach Golgatha! (1975) für Schlagzeug, Harfe und Orgelportativ. Die unten zur Sprache kommende Messe für Johannes XXIII (1968) fällt aus diesem Rahmen heraus. Darüber hinaus gibt es Werke, die nur indirekt auf eine religiöse Thematik hinweisen, sei es als Adaption einer Gebetsform (die Litaneien 1 bis 5, 1979-1982), als Sakralisierung eines musikalischen Intervalls (Vierzehn heilige Quinten mit Aureole, 1986), oder als Verbindung so disparater Elemente wie eines astrologischen und apokalyptischen Opernstoffs (Aquarius, 1983-1993). Aus alledem geht hervor, dass Goeyvaerts sein Verständnis des Katholizismus in ausgewählten Kompositionen aus der frühesten bis zur spätesten Schaffensphase auf unterschiedlichste Weise zum Klingen brachte.

Auch Karlheinz Stockhausen (1928-2007) entstammt einer katholischen Families. Wie eng Musik und Religion für ihn von Anfang an miteinander verbunden waren, geht aus einem Brief an Goeyvaerts hervor, der inmitten der Krise beim Komponieren von Spiel für Orchester geschrieben wurde: 22 Juli 1952; zitiert in: Karel Goeyvaerts: Selbstlose Musik. Texte - Briefe - Gespräche, hg. von Mark Delaere, Köln 2010, S. 267. 
Ich bin irre und weiß nicht, ob ich das Stück weiter schreiben soll. Wenn ich Dir das sage, wirst $\mathrm{Du}$ es wohl verstehen, aber mir nicht helfen können. Es ist eine unsagbare Not. Und ich weiß auch nicht, ob es recht ist, wenn ich aufhöre. Denn das, was ich da tue, ist so sehr etwas, was außer mir entsteht und sich selbst aufgegeben hat - als wollte es nur erfüllt sein; ohne mich, ohne einen Menschen zu befragen. Ich werde warten, und Gott bitten, dass er mich leitet und still sein lässt ${ }^{6}$.

Seine strenge Tagesdisziplin umschreibt er Anfang November 1953 wie folgt: „Früh aufstehen, zur Kirche gehen, dann der Tag über streng arbeiten und im Gebet einschlafen"7 . Katholische Praxis und ein Gespür für Transzendenz, das sich während der Ausbildungsjahre auch in seinem Interesse für Hermann Hesses Roman Das Glasperlenspiel manifestierte ${ }^{8}$, prägen Stockhausens Produktion aus den 195oer Jahren also entscheidend. Später bezog er zunehmend Aspekte östlicher Religionen und der Esoterik in sein musikalisches Denken ein, und trat aus der katholischen Kirche aus ${ }^{9}$. Der Austritt wurde sicherlich motiviert durch die Erweiterung des Gedankenhorizonts und das Interesse an alternativen Religionen und Lebensformen, die die 1960er Jahre prägten. Nach wie vor bleibt die Verbindung von Musik und irgendeiner Form von Spiritualität und Kosmologie für Stockhausen aber grundlegend, sei es in Form einer Rezitation von magischen Namen in Stimmung (1968), sei es durch das schwingende Verhältnis der Musiker zum Weltall in Aus den sieben Tagen (1968), sei es durch die Verbindung einer dem Werk zugrundeliegenden musikalischen Idee mit einer Gebetsformel in Mantra (1970) oder sei es in der szenisch-musikalischen Gestaltung von Gebetshaltungen in Inori (1973-74). Selbstverständlich ist diese Verbindung von der Stockhausen-Forschung umfangreich rezipiert worden, allerdings mit unterschiedlichen Schlussfolgerungen. Als sehr unterschiedliche Beispiele seien hier lediglich die Auffassung, bei Stockhausens Sakralität handele es sich um einen Super-Katholizismus, in dem sich unterschiedliche Inhalte wie Pantheismus und buddhistischer Atheismus zur Bedeutungslosigkeit verflüchtigten ${ }^{10}$, auf der einen und die Proklamation des Leidens als Fundament jeglichen geistigen Lebens auf der anderen Seite angeführt. Dieses Leiden mute Stockhausen seinen Hörern dann systematisch durch Anhäufung von Dissonanzen, die Bevorzugung von abstrakten Kompositionstechniken gegenüber freiem Ausdruck und durch die Integrierung von Unbestimmtheit und Steigerung der Komplexität zu ${ }^{11}$. Auch die Bedeutung des esoterischen Urantia-Buchs für die späteren Zyklen Licht (1977-2003) und

6 Brief vom 15. Februar 1952, in: Goeyvaerts - Stockhausen. Briefwechsel, hg. von Misch und Delaere (wie Anm. 1), S. 52.

7 Ebd., S. 154.

8 Dazu zuerst Herman Sabbe, Karlheinz Stockhausen ... wie die Zeit verging ... (Musik-Konzepte 19), München 1981, S. 64-68; dann ausführlicher Christoph von Blumröder, Die Grundlegung der Musik Karlheinz Stockhausens (BzAfMw 32), Stuttgart 1993, S. 9-27.

9 Thomas Ulrich, Neue Musik aus religiösem Geist. Theologisches Denken im Werk von Karlheinz Stockhausen und John Cage, Saarbrücken 2006, S. 245.

10 Clytus Gottwald, Neue Musik als spekulative Theologie. Religion und Avantgarde im 2o. Jahrhundert, Stuttgart 2003, S. 132-146.

11 Robin Maconie, „Saving the Faith: Stockhausen and Spirituality“, in: Tempo 72, 2017, S. 7-20. 

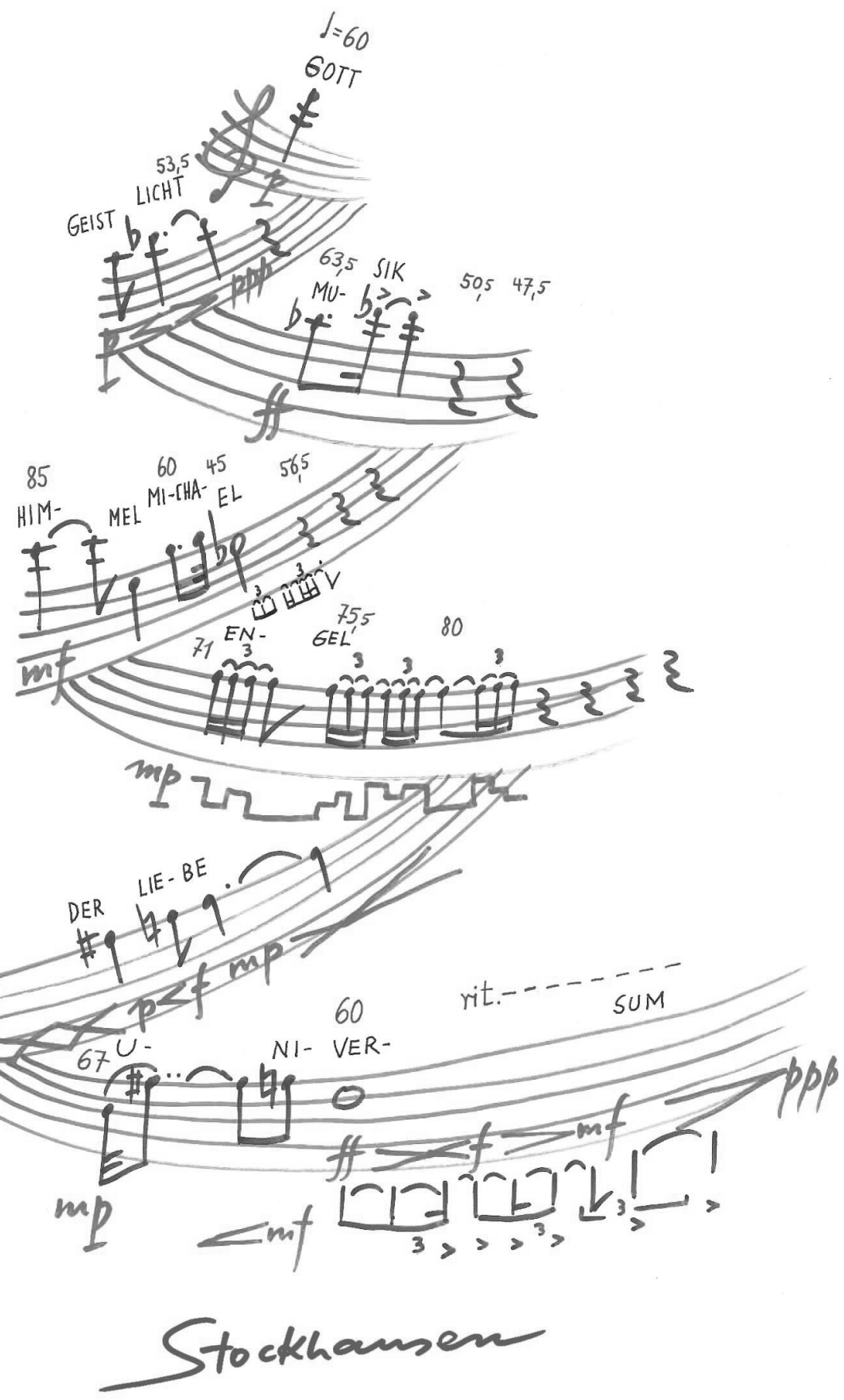

Abb. 1: Stockhausens Antwort auf ein Rundschreiben des Wochenblatts Die Zeit (Weihnachten 1991), das nach den „zehn wichtigsten Worten“ fragte. Mit freundlicher Genehmigung der Stockhausen Stiftung, Kürten. 
Klang (2004-2007) ist aufgearbeitet. ${ }^{12}$ Am ausführlichsten und eingehendsten ist die Bedeutung der Religiosität für Stockhausens Schaffen aber von Christoph von Blumröder und Thomas Ulrich erörtert, und zwar sowohl aus musikwissenschaftlichem als auch theologischem Blickwinkel. Anhand von Textdokumenten - allen voran anhand der Briefe an Hermann Hesse und Karel Goeyvaerts - und von Kompositionstechnik mit Gehalt verbindenden Werkexegesen - vor allem im Blick auf Kontra-Punkte und Klavierstück III - erforscht Blumröder die Ausbildung des musikalisch-religiösen Denkens Stockhausens in den Jahren 1948-1953, die er auch für das spätere Schaffen bis in den Opernzyklus Licht als grundlegend erachtet ${ }^{13}$. Ulrich betont eine Akzentverschiebung innerhalb des katholischen Paradigmas, indem er das Gottesbild des jungen Stockhausen vor allem auf die Idee einer perfekten metaphysischen Ordnung zurückführt, die es in der kompositorischen Arbeit nachzuahmen gelte. Ferner verbindet er die intuitive Musik mit ihrer Thematisierung von kosmischen Schwingungen mit Pfingsten und dem Konzept einer göttlichen Offenbarung durch feurige Zungen, um schließlich die (Multi-)Formel Kompositionen als Inkarnation einer kosmischen Ordnung in konkreter Gestalt zu interpretieren ${ }^{14}$. Stockhausens unablässige Bemühung, Musik, Kosmos und Gottesdienst aufeinander zu beziehen, lässt sich vielleicht am besten aus einem Bild erkennen (siehe Abb. 1 auf Seite 139).

Gott und Universum umfassen die Auflistung wie Alpha und Omega, die Zwölftonreihe schließt sich wie ein Kreis, die Pausen werden stufenweise länger, der letzte Abschnitt zeigt eine symmetrische Wiederholung der Schlusstöne, die sowohl das Intervall der perfekten Quinte wie der übermäßigen Quarte formen, und auch die rhythmischen, agogischen und dynamischen Abstufungen versuchen der göttlichen Ordnung gerecht zu werden.

\section{Die „geistlichen Grundlagen“ des Serialismus}

Die Bedeutung der Religiosität im Leben und Werk der beiden Komponisten dürfte aus obenstehender kurzer Übersicht klar sein. Kehren wir jetzt zum Schwerpunkt dieses Aufsatzes zurück, der Entwicklung und Ausführung einer seriellen Kompositionstechnik und Ästhetik während der ersten Hälfte der 1950er Jahre. Stockhausen und Goeyvaerts begegnen sich zum ersten Mal während der Darmstädter Ferienkurse von $1951^{15}$. Letzterer beschreibt in seinem Selbstporträt das Ereignis wie folgt:

12 Siehe u. a. Christian Ruch, „... but what I've read, I think, it's true. Karlheinz Stockhausen and The Urantia Book", in: The Musical Legacy of Karlheinz Stockhausen: Looking Back and Forward, hg. von Morag J. Grant und Imke Misch, Hofheim 2016, S. 148-157.

13 Blumröder, Die Grundlegung (wie Anm. 8).

14 Ulrich, Neue Musik aus religiösem Geist (wie Anm. 9).

15 Diese erste Begegnung und die Vorstellung des zweiten Satzes von Goeyvaerts' Nr. 1. Sonate für 2 Klaviere (1950-1951) in Th. W. Adornos Kompositionsseminar wurde des Öfteren und in den farbigsten Details beschrieben, z. B. in Kurtz, Stockhausen (wie Anm. 5), S. 57-61. 
Auf der Wiese der Marienhöhe wurde viel diskutiert. Wir hatten ein großes Bedürfnis nach Austausch, denn in der Nachkriegszeit arbeiteten wir noch alle mehr oder weniger isoliert. Es bestand großes Interesse an dem, was die anderen taten. Vom ersten Tag an zeigten wir uns gegenseitig unsere Partituren. Und sofort war zu spüren, dass der Serialismus in der Luft lag. Dennoch fanden meine Ideen über eine „statische Musik“ wenig Gehör - das musikalische Werk als eine Raum-Zeit-Projektion einer Grundidee betrachtet, welche die Struktur erzeugt. Mein parameterübergreifendes Prinzip der „synthetischen Zahl“ wirkte hier noch wie ein Hirngespinst. Nur ein junger Mann zeigte Interesse und fragte mich immer weiter aus: Karlheinz Stockhausen. Ich erinnere mich noch, wie er beim Mittagessen den anderen die „geistlichen Grundlagen“ meiner neuartigen Techniken erklären wollte. Ich hatte ihm alles in einem Gemisch aus Deutsch und Englisch erläutert, das er trotz meines Gestammels schnell begriffí.

In der darauffolgenden Korrespondenz zwischen den beiden Komponisten werden die drei in diesem Zitat angesprochenen Themen weiter entwickelt: das Konzept einer statischen Musik, die Suche nach absoluter Reinheit und die „geistlichen Grundlagen“, gemeint ist die theologische Rechtfertigung serieller Kompositionstechniken ${ }^{17}$.

Goeyvaerts strebt nach einer statischen Musik, die der Bewegungslosigkeit des Seins gleichkommt. Auch spricht er von der Allgemeinheit des Seins und von der absoluten Existenz des Einmaligen, die er in seiner Musik verkörpern möchte. Zwar bewegen sich seine Kompositionen unvermeidlich in Zeit und Raum, aber diese Bewegungen seien nur proportionale Vergrößerungen und Verkleinerungen des Absoluten und Einmaligen, das an sich statisch $\operatorname{se}^{{ }^{18}}$. Akustische Klänge, selbst wenn elektronisch transformiert, gerieten für Goeyvaerts bald außer Betracht für die Verwirklichung statischer Musik, da sie „vom Geistigen nicht regiert werden“ (Brief vom 12. November 1952). Folglich beabsichtigt er, ab dann nur noch elektronische Musik zu schreiben, und zwar „mit toten Tönen“ (Nr. 4 [Dezember 1952], in der vier unveränderliche, „bewegungslose“ Klänge ständig wiederholt, und nur die dazwischenliegenden Pausen seriell variiert werden) oder „mit reinen Tönen“ (Nr. 5 [1953]: mit Sinustönen). Auch Stockhausen verfolgt anfänglich die Idee einer statischen Musik. Im Brief vom 10. August 1951 an Goeyvaerts beschreibt er, wie sämtliche Klangparameter von Mosaike - einer Frühfassung von Kreuzspiel - in ein statisches Formprinzip gefügt werden, das mit dem Sein selbst und mit der Wahrheit gleichgesetzt wird:

Mir will es sehr klar erscheinen, dass es im Grunde einfach ist, sich für die Wahrheit zu entscheiden, wenn man sich erst um sie bemüht und sie erkannt hat. Und das, was wir den Zeitgeist nennen, ist unsere Wahrheit, ist die Wahrheit überhaupt; es ist das Sein selbst, das sich

17 Mark Delaere, „The Stockhausen-Goeyvaerts Correspondence and the Aesthetic Foundations of Serialism in the Early 1950s“, in: The Musical Legacy of Stockhausen, hg. von Grant und Misch (wie Anm. 12), S. 20-34.

18 Siehe z. B. Goeyvaerts' Briefe an Stockhausen von 25. Januar, 18. und 25. Mai, 1. November 1952 und 9. September 1953, in: Goeyvaerts - Stockhausen. Briefwechsel, hg. von Misch und Delaere (wie Anm. 1). Dazu auch Jan Christiaens, „Absolute Purity Projected into Sound. Goeyvaerts, Heidegger and Early Serialism“, in: Perspectives of New Music 41, 2003, H. 1, S. 168-179. 
von sich aus mit den Zeitläufen verwandelt, ohne sich je selbst zu verlassen. Und ich glaube sagen zu dürfen, dass erschreckend wenige unter den Komponisten die Wahrheit suchen. Die Sprache: das ist das Sein selbst, das ist als eine unverstellende die Wahrheit. Heute in einer Sprache sprechen, die an der Wahrheit vorbeigeht, die also tot ist, ein Scheinklang: mich dünkt, das heißt „dynamische Musik“ schreiben, romantische Musik im Sinne des historischen und Einzelbegriffes ${ }^{19}$.

Wenn solche metaphysischen Ansprüche zur Diskussion stehen, sind Kompromisse ausgeschlossen und ist das Ideal von absoluter Reinheit verpflichtend. Damit ist das zweite ästhetische Thema dieser Korrespondenz angesprochen ${ }^{20}$. In der Verwirklichung dieses Ideals ergibt sich aber bald ein Unterschied zwischen den beiden Komponisten. Wie gesagt, ist für Goeyvaerts ab 1952 nur die auf Sinustönen basierte elektronische Musik imstande, die „geistlichen Grundlagen“ mit absoluter Reinheit zu vergegenwärtigen. Demgegenüber verwirft Stockhausen die Instrumentalmusik nicht (Brief vom 7. Dezember 1952) und hegt zunehmend Bedenken gegen die Überzeugung, Vollkommenheit überhaupt in einer Komposition darstellen zu können. Goeyvaerts gesteht, dass eine absolute Perfektion vielleicht tatsächlich schwer realisierbar sei, hält aber daran fest, dass sie das anzustrebende Ideal sei:

Auch wenn uns die vollkommensten Klangapparate zur Verfügung stünden, bleibt die Wahrheit der Klangstruktur immer unbestimmbar, ja illusorisch, da sie nur bis zur vollkommeneren Wahrheit reicht, die alles Vorgehende aufklärt. Wichtig dabei ist, ob man weiter annimmt, dass Musik die so vollkommen [wie] mögliche Wiedergabe in Klang einer geistlichen Struktur sein soll21 .

Um dieser Idee treu zu bleiben, nimmt er eine Verneinung von Subjektivität, Ausdruck und Schönheit in Kauf:

Während Du all Deine Werke nach der Vollendung noch ändern könntest, so war es mir verboten. So wird Deine Musik immer lieblicher klingen, und auch menschlicher. Sie gehört Dir auch mehr. Mir gehört nichts. Weißt Du noch, wie Du im vorigen Herbst in Antwerpen warst, und wir zur Einsicht kamen, dass damals nur noch die Zeit uns als organisierbare Dimension zur Verfügung stand? Du konntest noch Kontra-Punkte schreiben. Für mich ist es Nr. 4 (mit toten Tönen) geworden, eine unmenschliche, grausame Musik, die mich aber in all ihrer Reinheit fasziniert ${ }^{22}$.

Sowohl das Konzept einer statischen Musik wie die Aufgabe, absolut einwandfreie „geistliche“ Musik zu schreiben, gehen letztendlich auf die starke religiöse Überzeugung

19 Goeyvaerts - Stockhausen. Briefwechsel, hg. von Misch und Delaere (wie Anm. 1), S. 7.

20 Delaere, „The Stockhausen-Goeyvaerts Correspondence“ (wie Anm. 17), S. 27-31.

21 Brief vom 30. März 1953, in: Goeyvaerts - Stockhausen. Briefwechsel, hg. von Misch und Delaere (wie Anm. 1), S. 117 .

22 Brief vom 4. August 1953, in: Goeyvaerts - Stockhausen. Briefwechsel, hg. von Misch und Delaere (wie Anm. 1), S. 140. 
beider Komponisten zurück. Die Bigotterie in dieser Korrespondenz zwischen zwei führenden Avantgardekünstlern ist zuweilen erstaunlich. So ist zum Beispiel die Rede von der heiligen Theresa von Lisieux, deren Todessehnsucht als Rechtfertigung für das Aufgeben des freien, künstlerischen Handelns herangeführt wird:

Das „Sein in Gott“, wozu wir bestimmt sind, bestand und besteht zu aller Zeit. Wir haben nur vorläufig, während unseres Lebens auf Erden, die Möglichkeit frei zu handeln, also unserem "Sein in Gott“ etwas entgegenzusetzen. Die Bedeutung des Todes ist der Verlust (oder besser: Das Befreit-Werden von) des freien Handelns. Gott hat in seiner großen Liebe aber die Möglichkeit geschaffen, uns jede Lust zum freien Handeln zu entnehmen während des Lebens. Das heißt nicht das tatsächliche Befreien vom freien Handeln, weil damit auch jede Verantwortlichkeit aufgehoben wäre, sondern ein Empfinden der Lust, Ihn selbst schaffen zu lassen an unserer Sache, also einen Grad, der sehr hoch sein kann, vom Erreichen des „Seins in Gott“. Wenn dieses Erreichen sehr weit geht, bedeutet der Tod kaum noch etwas. Die hl. Theresia von Lisieux, die man kurz vor ihrem Tode danach fragte, ob sie verlange, bald zu sterben, sagte: „Nein, ich verlange nichts mehr, weil der Tod mir kaum noch etwas ändert“. Ich glaube, jedem Menschen ist die Gnade gegeben, sein „sein in Gott“ zu einem ziemlich hohen Grad zu führen und dem irdischen Zustand also zu entwachsen ${ }^{23}$.

Identitätsverlust, Selbstverneinung und Weltentsagung gehen hier aus dem Gottesdienst (im wortwörtlichen Sinne) des katholischen Künstlers hervor. „Für die wichtigsten Botschaften werden die Gehorsamsten ausgewählt", schreibt Stockhausen am 4. Februar 1952 an Goeyvaerts. Wie sehr die metaphysisch-religiöse Auffassung von Musik im Allgemeinen und die Selbsteinschätzung des Komponisten als Sprachrohr des Göttlichen im Besonderen mit der romantischen Musikästhetik eines Wackenroder, Tieck oder Hoffmann zusammenhängt, einer Ästhetik, die in erster Instanz heftig angegriffen wird, mag als Paradox in dieser Ausprägung des Avantgardismus gelten ${ }^{24}$.

Zusammenfassend lässt sich sagen, dass Goeyvaerts ab der ersten Begegnung im Jahr 1951 Stockhausen nicht nur die aus der Verbindung von Weberns Handhabung der Zwölftonmusik und Messiaens parametrischem Denken hervorgehenden kompositionstechnischen Grundlagen des Serialismus vermittelte und am Beispiel seiner Nr. 1 (Sonate für 2 Klaviere) belegte, sondern ihn auch auf einen Bezug zwischen seriellen Kompositionstechniken und ästhetischen Prinzipien hinwies, die auf ausgesprochen katholischen Überzeugungen basierten. Während Stockhausen sich in der nachfolgenden Korrespondenz zunehmend von Goeyvaerts' Vorstellungen von statischer Musik und absoluter Reinheit distanzierte, blieben die auf der Konfession basierten „geistlichen Grundlagen“ des Serialismus für beide uneingeschränkt gültig.

23 Brief von Goeyvaerts vom 16. November 1953, in: Goeyvaerts - Stockhausen. Briefwechsel, hg. von Misch und Delaere (wie Anm. 1), S. 155 .

24 Für eine Besprechung weiterer Merkmale der Korrespondenz, die auf eine theologische Begründung von Denken und Schaffen beider Komponisten deuten, siehe Delaere, „The Stockhausen-Goeyvaerts Correspondence" (wie Anm. 17), S. 31-33. 


\section{Voraussetzungen für eine angewandte serielle und elektronische Musik}

Vor allem Goeyvaerts benötigte aber noch eine eingreifende gedankliche Entwicklung, ehe er sich überhaupt mit dem Konzept einer funktionalen seriellen und elektronischen Musik - und sei es auch für eine kirchenmusikalische Anwendung - versöhnen konnte. Dies hängt mit seiner strikten Auffassung von Musik als Vertonung einer statischen, ewigen Idee zusammen, die sich grundsätzlich nicht mit welcher Funktion auch immer verbinden lässt. Auch in dieser Hinsicht verfestigt sich bei Goeyvaerts eine romantische Haltung, die er eigentlich überwinden wollte. Funktionale und absolute Musik stehen hier einander gegenüber, obwohl Goeyvaerts die unterschwelligen ideologischen Aspekte seiner absoluten Musik keineswegs verkennt und durchaus verstärken möchte („die geistlichen Grundlagen“). Zu den Fremdkörpern, die die absolute Perfektion der zu bildenden seriellen Klangstruktur grundsätzlich ausschließen, gehören laut Goeyvaerts unbeherrschbare akustische Klänge (wie sie in der musique concrète Verwendung finden), unkontrollierbare und unzuverlässige Musiker (wie etwa in der Aufführung von Instrumentalmusik) und die Sprache (als Grundbestandteil vokaler Musik). Während die ersten zwei Einwände sich durch die Produktion elektronischer Tonbandkompositionen für die Liturgie beheben ließen, ist die Verwendung des Fremdkörpers Sprache für liturgische Zwecke fast unvermeidbar. Bis etwa Mitte der 195oer Jahre hielt Goeyvaerts wahrhaft serielle vokale Musik für unmöglich, oder wenigstes für unerwünscht. Daher seine Kritik an Boulez' Le marteau sans maître, das er sowohl klanglich - durch die Suggestion eines orientalischen Klangbilds - wie auch durch die Einbeziehung eines Texts als ein Exot, wortwörtlich eben als Fremdkörper, innerhalb der seriellen Musik ablehnend betrachtete. Wahrscheinlich hielt er auch das Gelingen von Stockhausens erster serieller Arbeit in Form einer Vokalkomposition für unmöglich, obwohl die Umwandlung von Mosaik in Kreuzspiel zwischen August und Oktober 1951 in dieser ersten, noch abtastenden Phase der Korrespondenz lediglich festgestellt wird ${ }^{25}$. Wie im vorigen Abschnitt erläutert, distanzierte Stockhausen sich allmählich von Goeyvaerts' Zwangsvorstellung von Musik als klingender Erscheinung von absoluter Wahrheit und Vollkommenheit, deren Konsequenzen er nicht ziehen wollte oder konnte. Seine Einstellung zur textbasierten seriellen Musik tritt im Gesang der Jünglinge (1955-56) hervor, in dem sowohl elektronische wie konkrete Klänge verwendet werden - letztere in Form einer Knabenstimme, die Textstellen aus dem alttestamentlichen Buch Daniels singt. Sie zeigt sich aber auch im Darmstädter Vortrag aus dem Jahr 1957 über „Musik und Sprache", in dem er Boulez' Textabhängigkeit in Le marteau sans maître und Nonos Textunverständlichkeit in Il canto sospeso seine eigene Vorgehensweise im Gesang der Jünglinge

25 Am 10. August 1951 stellt Stockhausen das Konzept von Mosaike für hohe Stimme und Klavier detailliert dar und fragt Goeyvaerts, ob dieser Titel geeignet sei. Am 17. August antwortet Goeyvaerts, Stockhausen müsse die Titelfrage selbst entscheiden, und am 4. September, dass er über den beabsichtigten Besuch Stockhausens im Oktober sehr erfreut sei, damit sie über Mosaike und seine Nr. 2 reden könnten. Am 13. September berichtet Stockhausen, dass er in Mosaike noch eine Männerstimme und einen Schlagzeuger hinzugefügt, und am 22. Oktober, dass er mit dem Schreiben von Kreuzspiel angefangen habe (GoeyvaertsStockhausen. Briefwechsel, hg. von Misch und Delaere [wie Anm. 1], S. 5-24). 
gegenüberstellt, in dem sieben Stufen von Textverständlichkeit seriell variiert, und Vokale und Konsonanten zu Sinustönen und Geräuschen in Beziehung gesetzt werden ${ }^{26}$. Bei der ersten Begegnung 1951 in Darmstadt hatte Theodor W. Adorno Goeyvaerts und Stockhausen in Bezug auf Thomas Manns Roman Doktor Faustus als „Adrian Leverkühn und sein Famulus" charakterisiert. Angesichts ihrer gleichen Auffassung von den „geistlichen Grundlagen“ der seriellen Musik, aber auch ihrer unterschiedlichen Ausarbeitungen dieser Idee und ihrer divergierenden Auffassungen serieller Vokalmusik, wäre auch eine Kennzeichnung beider Komponisten in der ersten Hälfte der 1950er Jahre als Moses und Aaron nicht fehl am Platz: Goeyvaerts, der sich von der Reinheit des Denkens, der Unwandelbarkeit des Gesetzes und der Unvorstellbarkeit von Gott leiten lässt und dem schlussendlich das Wort fehlt; Stockhausen, der mit ansprechenden, teilweise auch vokalen Kompositionen seine Hörer beglückt ${ }^{27}$.

Es geht aus dieser Sachlage hervor, dass die nötige Gesinnungsänderung, um überhaupt zu einer kirchenmusikalischen Anwendung der seriellen und elektronischen $\mathrm{Mu}$ sik zu kommen, für Goeyvaerts viel grösser als für Stockhausen war. Interessanterweise finden sich die ersten Spuren einer konzeptuellen Wandlung bei Goeyvaerts im Kontext der Produktion eines elektronischen Werkes. Es handelt sich dabei nicht um Nr. 4 mit toten Tönen, deren Reinschrift im Dezember 1952 abgeschlossen wurde, deren Realisierung aber erst viel später, Anfang der 1980er Jahre, im IPEM Studio in Gent erfolgte. Die erste tatsächlich realisierte elektronische Komposition war demnach die nachfolgende, zwischen Januar und Juni 1953 komponierte Nr. 5 mit reinen Tönen. Beide Werke entstanden ohne jegliche Studio-Erfahrung seitens Goeyvaerts, der sich während seiner Studienzeit in Paris aus ästhetischen Gründen fern von Pierre Schaeffers elektronischem Musikstudio im RTF hielt und nach seiner Rückkehr in die Heimat, trotz vieler Anfragen beim belgischen öffentlichen Rundfunk NIR, nicht einmal die Möglichkeit hatte, sich elektronische Klänge anzuhören. Daher die vielen Fragen über das akustische Verhalten elektronischer Klänge zueinander und über die Bildung von Klangspektren aus der Überlagerung von Sinustönen in der Korrespondenz mit Stockhausen aus dieser Peri-

26 Karlheinz Stockhausen, „Musik und Sprache I/II/III“, in: Texte 2, Köln 1964, S. 58-68 und S. $149-166$. Dazu auch Pascal Decroupet, Inge Kovács, „Sprachkomposition“, in: Im Zenit der Moderne. Die Internationalen Ferienkurse für Neue Musik Darmstadt 1946-1966 2, hg. von Gianmario Borio und Hermann Danuser, Rombach 1997, S. 302-332, und Martin Iddon, New Music at Darmstadt: Nono, Stockhausen, Cage, and Boulez, Cambridge 2013, S. 147-153. Bemerkenswert ist das Vorwort zu „Musik und Sprache I“, in dem Stockhausen nicht nur die Textwahl sondern auch die Textbehandlung mit der ideologischen Gesinnung der Komponisten verbindet: „Abgesehen von nationalen, psychologischen und bildungsmäßigen Verschiedenheiten liegt ein wesentlicher Gegensatz darin begründet, dass die drei Musiker nicht dasselbe glauben. Für jeden von ihnen sieht die Wirklichkeit - das Leben - anders aus. Und das geht aus ihrer Musik hervor". Dem fügt Stockhausen etwas später noch „,zugespitzte Unterscheidungen wie zwischen Atheisten, idealistischen Kommunisten und Metaphysikern“ zu (Stockhausen, „Musik und Sprache I“, Zitate S. 149).

27 Selbstverständlich spiele ich auf Arnold Schönbergs Oper Moses und Aron (1930-32) an, auch wenn die Uraufführung des Tanzes um das goldene Kalb am 2. Juli 1951 in Darmstadt Goeyvaerts bitterlich enttäuschte („Hier matin j'ai été à une répétition du Veau d'or de Schönberg. C'est pire que Le dernier survivant de Varsovie! C'est du Verdi sériel! Le pauvre Schönberg doit être complètement gaga“; Brief vom 29. Juni 1951 an seinen Pariser Freund Jean Barraqué, in: Goeyvaerts: Selbstlose Musik, hg. von Delaere [wie Anm. 4], S. 284). Schließlich stellt diese Szene die Apotheose von Aaron dar. 
ode, daher auch das Beharren auf der Verwendung eines „stehenden Magnetophons“, eine Idee, die lediglich einer abstrakten Vorstellung statischer Musik entspringen konnte, jenseits von jeder praktischen Realisierungsmöglichkeit. Ab Juni 1953 wurde Stockhausen Mitarbeiter im Studio für Elektronische Musik des WDR Köln, unter Leitung von Herbert Eimert. Daher ergab sich für Goeyvaerts die Möglichkeit, im Januar 1954 nach Köln zu reisen, um dort Nr. 5 zu realisieren. Das Ergebnis war erschütternd, wie er in einem etwas später entstandenen, bezeichnenderweise „Zum Relativismus“ übertitelten, handgeschriebenen Text (1955) feststellt:

Bei der Realisation meiner Komposition Nr. 5 im Kölner Studio haben sich bestimmte Hörphänomene herausgestellt, mit denen man unbedingt rechnen sollte. Dabei ist mir eine Kluft zwischen abstrakter Strukturbildung und psycho-physiologischer Wahrnehmung aufgefallen. Diese Kluft war die größte Gefahr in einer Zeit, in der das musikalische Schaffen wie nie zuvor rationalisiert worden ist ${ }^{28}$.

Goeyvaerts beobachtet erstens, dass die gedachten rationalen Proportionen von Zeit, Lautstärke und Tonhöhe von der Dominanz der Tonhöhe in der Wahrnehmung durchkreuzt werden und ihr Sinn deshalb unhörbar wird; zweitens, dass reine Zeitwahrnehmung ohne Zeitmaß niemals absolut ist und die Zeitstruktur besonders in ihrer Beziehung zu den Verhältnissen anderer Dimensionen fast völlig verloren geht (insbesondere bei den in Nr. 5 häufig begegnenden längeren Zeitwerten); und drittens, dass die Lautstärke, die sich an und für sich so präzise in Dezibel komponieren lässt, in der Wahrnehmung unglaublich kontextabhängig ist ${ }^{29}$. Darum fügt er in seiner im Frühjahr 1954 komponierten Nr. 6 180 Klanggegenstände von gleicher Dauer und Lautstärke, die dadurch also noch immer die Idee einer statischen Musik vertreten, zu Tonkomplexen zusammen, die für das Ohr wie ein geschlossenes Klangfeld wahrnehmbar sind:

Nur musikalische Intuition und traditionelle Tonempfindung haben bei der Zusammensetzung dieses Feldes eine Rolle gespielt. Mir kommt es so vor, als ob solch ein empirisches Verfahren jetzt vor allen Dingen zur auditiven Zweckmäßigkeit der Komposition führt.

Bedeutend ist, dass Nr. 6 zum ersten Mal wiederum als Instrumentalmusik konzipiert wurde, eine Entscheidung, für die er Stockhausen kurz zuvor noch scharf kritisierte ${ }^{30}$. Dies ermöglicht, den Ablauf der Musik ständig der besonderen Situation des Augenblicks anzupassen:

28 Karel Goeyvaerts, „Zum Relativismus“, in: Goeyvaerts: Selbstlose Musik, hg. von Delaere (wie Anm. 4), Zitat S. 156.

29 Ebd.

30 Brief vom 26. Januar 1954, in Bezug auf Stockhausens Klavierstücke $V-X$ : „Und Du, Du kommst einfach, ohne weitere Bedenken wieder zum alten Klavier [zurück]. Mir erscheint das furchtbar!“, in: Goeyvaerts Stockhausen. Briefwechsel, hg. von Misch und Delaere (wie Anm. 1), S. 167. 
Dazu ist zu bemerken, dass der Mensch, als zeitliches Wesen, nur zu relativer Wahrnehmung imstande ist. Ein vorher geplanter Augenblick ist zwangsläufig von der Zeitsituation beeinflusst, in die er integriert wird. Bekommt er seine richtige Stellung im Zeitablauf zugewiesen, erweist der Plan sich meistens als partiell inadäquat. Deswegen redet man von der Einmaligkeit der Zeit. Das erklärt auch, warum die „Interpretation“ eines Stücks immer noch wichtig bleibt. Sie ist noch, was sie immer war, nämlich eine Vermittlung zwischen komponierter, also einmalig geplanter Musik, und der spezifischen Situation ihres Erklingens ${ }^{31}$.

Zwei Folgerungen drängen sich nach der Lektüre dieses Essays auf. Einmal mehr wird entgegen weit verbreiteter, vulgärer Auffassungen - die Bedeutung der Wahrnehmung in der Entwicklung der seriellen Komposition bestätigt ${ }^{32}$. Zwar geht Goeyvaerts bei der Konzeption einer Komposition statt von einer Klangvorstellung noch immer von einer abstrakten, metaphysischen bzw. theologischen Grundidee aus, aber wenn diese Idee im Hörerlebnis nicht erfahren und von zahlreichen psychophysiologischen Phänomenen entstellt wird, sollte der Komponist zwischen abstrakter Strukturidee und Wahrnehmung vermitteln. Die zweite wichtige Folgerung bezieht sich eben auf diese Vermittlung, für die Goeyvaerts bis dahin von ihm verpönte Konzepte wie Relativismus, musikalische Intuition, traditionelle Tonempfindung, empirisches Verfahren, freie Verwendung und Interpretation anführt. Stockhausen hatte ihn bereits Ende 1952, im Kontext einer Diskussion über das schon erwähnte stehende Magnetofon, auf die Unvermeidbarkeit einer solchen Vermittlung hingewiesen:

Ich möchte Dich doch daran erinnern, dass gewiss nichts vollkommen homogen, vollkommen statisch, dass nichts außer der Zeit sein wird auf dieser Welt. Natürlich hat die Dimension des Bandes nichts essentielles (Du sagst fundamentelles) mit der Frage des Zeitablaufes (oder nicht) zu tun. Das existentielle Homogen-Sein ist nicht einmal in der Stille, viel weniger bei einem Klang zu erreichen. [...] Wenn das aber so ist, bleibt für uns Musiker nur die Möglichkeit des relativen Homogen-Seins, das auf den praktisch erfahrenden Geist, auf Sinneswahrnehmung und deren Verarbeitung gerichtet ist. Das Absolute bleibt uns unerreichbar, denn alles „Klingen“ ist an Zeit gebunden. Deine Unterscheidung ist abstrakt philosophisch (laufendes - nicht laufendes Band = relatives - absolutes Homogen-Sein). [...] Ich meine, die Grenze des akustisch Wahrnehmbaren dürfte dem musikalischen Gestaltungswillen ausreichen. Das Geistige geht immer weiter. Das Wesen der „Transzendenz“ ist der Zeit verhaftet. Drüben gibt es keine Transzendenz, aber auch keine Musik mehr. ${ }^{33}$ Der Zusammenhang von Theorien über musikalische Zeit, Wahrnehmung und seriellen Kompositionstechniken ist einsichtig beschrieben in Gianmario Borio, „Kompositorische Zeitgestaltung und Erfahrung der Zeit durch Musik. Von Stravinskys rhythmischen Zellen bis zur seriellen Musik“, in: Musik in der Zeit, Zeit in der Musik, hg. von Richard Klein, Eckehard Kiem und Wolfram Ette, Weilerswist 200o, S. 313-332. Anm. 1), S. 99. 
Fest steht, dass die selbst beobachtete „partielle Unzweckmäßigkeit“ der musikalischen Struktur von Nr. 5 und mehr noch, das völlige Scheitern von der unvollendet gebliebenen Nr. 7 mit konvergierenden und divergierenden Klangniveaus, einer Tonbandkomposition, die Goeyvaerts unter Mitarbeit von Gottfried Michael Koenig in April 1955 im Kölner Studio zu realisieren versuchte, Goeyvaerts in eine tiefe künstlerische und auch menschlich-existentielle Krise stürzte. Fest steht aber auch, dass dadurch die Bedingungen, um über eine funktionale serielle und elektronische Musik für kirchenmusikalische Zwecke überhaupt nachzudenken, auch für Goeyvaerts erstmal geschaffen waren.

\title{
Gesang der Jünglinge
}

Gleich am Anfang ihres Briefwechsels spricht Stockhausen die Spannung zwischen fortschrittlichem Komponieren und katholischem Konservatismus an:

\begin{abstract}
Mir ist es sehr darum zu tun, wie Du siehst, in allem, was ich mache, sehr klar das Geschehen herauszukristallisieren, also immer das Material ganz zu bewältigen und der Idee zu unterstellen. Wie anders ist das mit mir geworden! Ich bin sehr froh darüber und sehe meinen Weg. Es ist mir, als sei ich in allem Gott näher gekommen; und das ist das beste. - Wir sind in unserem Wesen als Katholiken konservativ. Das gründet in der hierarchischen Fügung. Wie sehr es in meinem Leben den scheinbaren Widerspruch zwischen jenem Konservatismus und einer Sehnsucht nach dem Zukünftigen, dem Neuen oder auch Urzeitlichen zu lösen gibt, spüre ich immer mehr. Es wird die Aufgabe sein. Das ist in unserem abendländischen Raum das Lutherproblem, das geistige Problem der Neuzeit überhaupt und vor allem eben das künstlerische Problem ${ }^{34}$.
\end{abstract}

Gut vier Jahre später scheint sich der scheinbare in einen realen Widerspruch zu wandeln und Stockhausen mit der hierarchischen Struktur des Katholizismus kollidiert zu haben. Der Stockhausen-Biograf Michael Kurtz erörtert, dass Stockhausen, nach dem Konzert mit im Kölner Studio realisierter elektronischer Musik am 19. Oktober 1954, den Plan gefasst habe, eine Messe für Stimmen und elektronische Klänge zu komponieren, von der er hoffte, dass sie im Kölner Dom uraufgeführt werden könnte. Laut Kurtz sollte Herbert Eimert sich dafür beim erzbischöflichen Generalvikariat eingesetzt, jedoch mit der Begründung, „Lautsprecher gehören nicht in eine Kirche“, einen abschlägigen Bescheid bekommen haben, weshalb der herb enttäuschte Stockhausen das Konzept einer elektronischen Messe in ein (nicht-liturgisches) geistliches Werk umgesetzt habe, eben den 1955-56 komponierten Gesang der Jünglinge ${ }^{35}$. Da sich im Stockhausen Archiv keine Unterlagen befinden ${ }^{36}$, die auf einen Kontakt mit den zuständigen kirchlichen BehörAnm. 1), S. 7.

35 Kurtz, Stockhausen (wie Anm. 5), S. 116-117.

36 Laut Mitteilung von Maria Luckas an den Verfasser am 2. Juli 2018. Auch Skizzen oder auch nur Hinweise zu einer geplanten Messe fehlen im Archiv. 
den schließen lassen, geht diese Information wahrscheinlich auf Gespräche von Kurtz mit Stockhausen zur Vorbereitung seiner Biografie zurück. In seinen öffentlichen Äußerungen über den Gesang der Jünglinge erwähnt Stockhausen den ursprünglichen Plan allerdings nicht, weder im Programmtext zum Konzert am 30. Mai 1956, noch im Artikel Aktuelles für die Reihe 1 (1955), der ausschließlich dieser Komposition gewidmet ist, und auch nicht im Vortrag „Musik und Sprache III“ bei den Darmstädter Ferienkursen 1957, der später in die Reihe 6 (1958) veröffentlicht wurde ${ }^{37}$. Stattdessen bietet er dem Leser in beiden letztgenannten Texten einen gründlichen Einblick in die ästhetischen und kompositionstechnischen Voraussetzungen, die die Ausarbeitung dieses Werks geleitet haben. Zusammen mit der maßgebenden, auch auf Skizzenstudien basierten Analyse von Pascal Decroupet und Elena Ungeheuer ${ }^{38}$ sind die wichtigsten analytischen Tatsachen über diese Komposition aufgeschlüsselt, sodass eine musikanalytische Besprechung im Kontext dieses Artikels sich erübrigt.

Über die Vorgeschichte des Gesang der Jünglinge erschien 2009 aber noch ein Beitrag, der die Darstellung von Kurtz zu korrigieren oder wenigstens zu nuancieren beabsichtigte ${ }^{39}$. Von der Kurtz'schen „Legende“ behält Helmut Kirchmeyer lediglich die Feststellung bei, Stockhausen habe das neue Medium der elektronischen Musik symbolhaft mit einer ästhetisch anspruchsvollen Missa solemnis beginnen lassen wollen, für deren Uraufführung er aber keine ausdrückliche Zusicherung eines Sakralraums oder eines Gottesdienstes bekommen habe, weshalb er diesen Plan schließlich aufgegeben habe. Aufgrund der Nachfragen von Reinhard Pabst bei dem damaligen Referenten für Kirchenmusik im Erzbistum Köln im Jahr 1982 stellt Kirchmeyer aber fest, dass dazu nie eine offizielle Anfrage, weder von Stockhausen noch von Eimert, an das dafür nicht einmal befugte Generalvikariat gerichtet wurde, und dass auch Johannes Overath, der als Präses des Allgemeinen Cäcilienverbandes in Deutschland, Professor für Kirchenmusik aber auch Mitglied des Kölner Rundfunkrates, hier hätte vermitteln können, zu keinem Zeitpunkt mit dem Thema "Elektronische Messe“ auch nur inoffiziell befasst war $^{40}$. Eine offizielle Ablehnung seitens einer kirchlichen Behörde hat es daher nicht gegeben, Kirchmeyer suggeriert aber, dass Eimert die Risiken von Stockhausens Vorhaben für allzu groß einschätzte, und ihn deshalb in irgendeiner Form an eine Ablehnung glauben ließ:

Eimert musste sich ausrechnen, dass die Wahrscheinlichkeit eines künstlerischen Durchbruchs, gesehen aus der Zeit vor dem Gesang, äußerst gering, die eines Misserfolges dagegen umso größer war. In der Konsequenz hätte das bei Ablehnung zu einer Missstimmung, bei Unterstützung aller Voraussicht nach zu einer Schwächung Overaths und mit der Schwächung des Bundesgenossen im Rundfunkrat zu einer solchen der eigenen ohnehin gefährdeten Posi-

Diese drei Texte sind in Stockhausen, Texte 2, S. 49-68, aufgenommen.

Pascal Decroupet und Elena Ungeheuer, „Through the Sensory Looking-Glass: the Aesthetic and Serial Foundations of Gesang der Jünglinge", in: Perspectives of New Music 36, 1998, H. 1, S. 97-142.

Helmut Kirchmeyer, „Stockhausens Elektronische Messe nebst einem Vorspann unveröffentlichter Briefe aus seiner Pariser Zeit an Herbert Eimert", in: AfMw 66, 2009, H. 3, S. 234-259.

Ebd., S. 254-255. 
tion geführt. Also brachte Eimert, schon immer ein hervorragender Kenner von Personen und Sachlagen, mit denen er es zu tun hatte, Overath gar nicht erst ins Spiel, und wir wissen, dass es die anderen Rundfunkleute, vermutlich aus demselben Grund, auch nicht taten. Was Eimert, sollte die Version Pabsts richtig sein, hinterher Stockhausen erzählte, um seine Ruhe zu haben, was er wem zuschob, um seinen in dieser verqueren Situation widerstreitender Interessen hilfreichen innenpolitischen Freischlag zu kaschieren und den tatsächlichen Erfolg nicht zu gefährden, bleibe dahingestellt ${ }^{41}$.

Dieser Aufsatz zielt nicht so sehr auf die Frage, wer für das Scheitern des ursprünglichen Vorhabens verantwortlich war, sondern vielmehr auf das Konzept einer angewandten seriellen und elektronischen Musik, wie es Stockhausen damals vorschwebte und wie er es in einer Messe paradigmatisch realisieren wollte. In der Korrespondenz mit Goeyvaerts lässt sich darüber noch einiges finden. Die Messe wird zum ersten Mal in einem Brief von Goeyvaerts (29. Dezember 1954) erwähnt: „Hast Du mit Deiner Messe angefangen?". In den erhaltenen Briefen von Stockhausen war davon zuvor nicht die Rede, sodass Goeyvaerts diese Frage wohl nur aufgrund eines verlorengegangenen Briefes oder eines mündlichen Austauschs mit Stockhausen, vielleicht schon während Goeyvaerts' Kölner Aufenthalt im Januar 1954 oder im Kontext des Kölner Konzertes mit elektronischer Musik im Oktober 1954, stellt. Allerdings beantwortet Stockhausen diese Frage ausführlich in einem undatierten Brief, der aus inhaltlichen und kontextuellen Gründen aber bestimmt Ende Januar oder Anfang Februar 1955 geschrieben wurde:

Elektr. habe ich noch nichts wieder angefangen. Die Schwierigkeiten übersteigen das Maß des Erwarteten. Es wird keine Messe sein. Die kirchliche Behörde lehnt so etwas von vorneherein ab. Ich habe mich erkundigt. Ich denke aber an die Funktion vor allem, wenn ich so etwas machen würde. Und da scheint zum jetzigen Zeitpunkt etwas nicht in Ordnung zu sein. Nun bin ich entschlossen, den „Gesang der Jünglinge im Feuerofen“ (Benedicite) zu komponieren. Mich drängt es sehr danach, in aller jetzigen Schwierigkeit des Materials und des Handwerklichen mich selbst an einen so glücklichen, zeitlosen Text des Gotteslobes zu binden - und das Unbestimmbarste, die Menschenstimme, mit dem Bestimmtesten, der elektr. Materie, zu vereinen ${ }^{42}$.

In dieser Mitteilung tritt Stockhausens Absicht, Funktionsmusik für die katholische Liturgie zu schreiben, klar hervor („Ich denke an die Funktion vor allem“). Eine innerhalb oder außerhalb eines Kirchengebäudes aufgeführte Konzertmesse, etwa in der Tradition Beethovens, kommt ihm nicht in den Sinn. Stattdessen wollte er elektronische Musik produzieren, die als Ausprägung der kanonischen Gebete in den liturgischen Verlauf integriert ist. Aufgrund der damals geläufigen Tridentinischen Liturgie

41 Ebd., S. 257-258. Trotz des vielversprechenden Titels bleibt der Aufsatz von Ross Wallace Chait, „Gesang Dream: Functions of Faith in Stockhausen's Electronic Mass“, in: Perspectives of New Music 52, 2014, H. 3, S. 185-196, hinter dem (damaligen) Kenntnisstand weit zurück. 
erscheint es naheliegend, dass Stockhausen vorhatte, die lateinischen Texte des Ordinariums als Textgrundlage zu verwenden. Sobald das Projekt sich aber als unausführbar erwies, veränderte Stockhausen die Funktion von liturgischer Kirchenmusik zu Konzertmusik ${ }^{43}$. Zwar begegnet das „Benedicite“ aus dem alttestamentlichen Buch Daniël $(3,52-90)$ auch in der katholischen Gebetspraxis, entweder als Teil des Stundengebets der „Liturgia Horarum“ von kontemplativen Klostergemeinschaften und Priestern oder als Lobgesang nach der Messe. Im letzteren Falle ist es aber kein fester Bestandteil der Messliturgie, sondern ein Zusatz, der also, wie inzwischen das „Magnificat“, eher in einem ästhetischen als einem sakralen, liturgischen Kontext aufgeführt wird. Gesang der Jünglinge ist deshalb ein Konzertwerk, eine „Opusmusik“, deren religiöser Gehalt sich lediglich in den obersten, primär semantischen der sieben Textverständlichkeitsgraden zeigt $^{44}$.

Wie aus Stockhausens Vortrag und aus dem Aufsatz von Decroupet und Ungeheuer hervorgeht, hat Stockhausen sich sehr bemüht, ein Farbkontinuum zwischen den gesungenen und den elektronischen Klängen zu schaffen. Damit saugt er sozusagen die konkrete in die serielle elektronische Musik und Ästhetik auf. In der Erläuterung seines Vorhabens an Goeyvaerts in dem vorher erwähnten Brief (Ende Januar oder Anfang Februar 1955) mag er sich an dessen Abneigung gegen konkrete Musik im Allgemeinen und textbasierte serielle Musik im Besonderen erinnert haben. Deswegen bemüht er sich extra, den Gebrauch einer unbestimmbaren, für Goeyvaerts also unvollkommenen Menschenstimme im Gesang der Jünglinge aus der Hörerfahrung zu rechtfertigen:

Denn wenn man es einmal ganz tief erlebt hat, machen nur die verschiedenen Haltungen der Komponisten einen Unterschied von „chaotisch“ und „streng geordnet“. Den gibt es aber nicht, wenn man der „Gedankenordnung“ Ordnungskriterien und Ordnungsmethoden der Empfindungsqualitäten hinzufügt. Wenn man ganz konsequent erforscht hat, dass keine gedankliche Wahrnehmung von Musik (und ebenso keine unbewusste) existiert, die nicht über das Ohr mit allen Folgerungen geht...45

43 Entgegen geläufiger Meinungen ist elektronische Musik keine „Radiomusik“, sondern Konzertmusik. In einer Radiosendung fehlt die für den Gesang der Jünglinge wesentliche räumliche Dimension. Auch die Reduktion von fünf (oder vier) auf zwei Kanäle für eine stereofonische Schallplattenproduktion ist ein unbefriedigender Kompromiss.

44 Stockhausen, „Musik und Sprache III“, S. 60: „Dem ,Farb'-Kontinuum entsprechend ging die Komposition von der Vorstellung eines ,Sprachkontinuums‘ aus: gesungene Lautgruppen sind an bestimmten Stellen der Komposition zum verständlichen Sprachzeichen, zum Wort geworden, zu anderen Zeitpunkten bleiben sie reine Klangqualitäten, Lautzeichen; und zwischen diesen Extremen gibt es verschiedene Grade der Textverständlichkeit“. 


\section{Messe für Johannes XXIII}

Ausgehend von den in „Zum Relativismus“ dokumentierten Erfahrungen mit Nr. 5 und Nr. 6 während des ersten Halbjahrs von 1954, hatte auch Goeyvaerts zu dieser Zeit Denken und Hören in seiner Auffassung serieller Musik ins Gleichgewicht gebracht, und die Verwendung von „konkreten“ Instrumentalklängen nicht länger zurückgewiesen. Dass die Auflockerung sich schließlich auch auf Vokalklänge und deren Gebrauch in angewandten kirchenmusikalischen Kompositionen ausdehnen sollte, geht aus seiner Korrespondenz mit Ambroos Verheul (1916-2005) über eine neue, zeitgemäße Gestaltung der Liturgie hervor. Dieser Benediktinermönch aus der Abtei zu Affligem war ab 1946 als Sekretär, ab 1964 als Schriftleiter der Tijdschrift voor Liturgie, ab 1956 als Veranstalter der jährlichen liturgischen Tagungen und ab 1966 als Herausgeber der internationalen Zeitschrift Questions Liturgiques - Studies in Liturgy Mittelpunkt der Liturgiereform nicht nur im belgischen Katholizismus ${ }^{46}$. Hauptthema der ersten liturgischen Tagung in November 1956 in der Norbertiner Abtei zu Tongerlo war die theologische Rechtfertigung der aktiven Teilnahme der Gemeinde während der Eucharistiefeier. Die zweite Tagung in Oktober 1957 in der Affligemer Abtei war der Liturgie der Taufe und der Seelsorge gewidmet. Die Tagungsprogramme beabsichtigen nachdrücklich, die hierarchische, distanzierte Tridentinische Liturgieform durch eine modernere zu ersetzen; in dieser Hinsicht können sie als eine Vorbereitung auf das Zweite Vatikanische Konzil (1962-1965) aufgefasst werden. Goeyvaerts hat an diesen Tagungen zwar nicht teilgenommen, war aber von seinem Bruder, dem Priester, über die Absichten der ersten Tagung aufgeklärt worden und kontaktierte daher Verheul zum ersten Mal Anfang Dezember 1956. Kurz danach lädt er Stockhausen ein, an dieser Reflexion über die Funktion von Musik bei der Gestaltung neuer Liturgieformen teilzunehmen ${ }^{47}$.

Der Brief an Verheul enthält Goeyvaerts' erste Ideen zu einer seriellen liturgischen Musik. Er beginnt mit der Feststellung, dass der gregorianische Choral bis etwa 1600 das Wesen der Kirchenmusik verkörperte, sich danach aber weder als selbständige Form noch als Grundlage polyfoner Messkompositionen weiterentwickelt habe und deshalb heute nicht länger als stilistisches Modell dienen könne. Goeyvaerts argumentiert, dass aus der Verbindung von Tradition und Erneuerung eine Alternative entwickelt werden sollte. Hier sei man daran erinnert, dass Stockhausen in einem seiner ersten Briefe an Goeyvaerts das angedeutet hatte, was er als das geistige Problem der Neuzeit ansah, nämlich der scheinbare Widerspruch zwischen katholischem Konservatismus und einer Sehnsucht nach dem Zukünftigen (siehe oben). Für die musikalische Gestaltung der Liturgiereform stellt Goeyvaerts der „heutigen individualistischen religiösen Kunst“ eine bert Beauduin (1873-1960), war ein Anfang gemacht mit einer auf Verständlichkeit und Gemeindebeteiligung zielenden Liturgiereform, die sich dann in den 1950er Jahren intensivierte.

47 Siehe der in der Einführung dieses Beitrags zitierte Brief vom 22. Dezember 1956. Goeyvaerts' Einladung erfolgt selbstverständlich aufgrund von Stockhausens (gescheitertem) Projekt einer Messe für Stimmen und elektronische Klänge. 
Alternative gegenüber, in der mittelalterliche künstlerische Auffassungen und Praktiken mit zeitgenössischen Materialien verknüpft werden:

\begin{abstract}
Wir sind auf musikalischem Gebiet jetzt so weit, dass eine Klangstruktur durch Gemeinarbeit erzeugt werden kann, ganz im Sinne einer kollektiven Handwerkerschaft (mit dem Vorgehen der Kathedralbauer vergleichbar). Dieser Klangstruktur sollte aber auf strikt liturgischen Fakten basieren, damit sie persönlichen Interpretationen enthoben bleibt. Und hier brauche ich also Ihren Rat: Halten sie eine solche Zusammenarbeit von Liturgist, Tonsetzer und Tontechniker für erwünscht? Hier spreche ich auch im Namen einiger ausländischen Kollegen, die ebenfalls dafür eifern möchten. Sie werden sich wahrscheinlich fragen, warum ich Derartiges vorschlage? Allererst, weil ich die Musik, wie sie jetzt geworden ist, für liturgische Zwecke geeignet erachte. Daneben auch, weil sich jetzt die Aufgabe stellt, für die Radio- oder FernseheMesse eine Form zu finden - insofern Sie es natürlich nicht für untauglich halten, die Liturgie außerhalb ihres eigenen, kirchlichen Rahmen zu bringen ${ }^{48}$.
\end{abstract}

Da Goeyvaerts damals völlig isoliert lebte und arbeitete ${ }^{49}$, sind „die ausländischen Kollegen“ sicherlich auf Stockhausen beschränkt. Auffallend ist natürlich der erwähnte Medienwechsel zu Radio- und Fernsehe-Messen, letztere erst drei Jahre nach Anfang der Fernsehübertragungen in Belgien. In mehreren Hinsichten ist das präkonziliäre sakrale Ritual der seit dem 19. Jahrhundert üblichen Konzertpraxis vergleichbar: An bestimmten, dazu geeigneten Ort und Zeitpunkt sammeln sich Leute, um festgelegten (Musik)texten andächtig zuzuhören und rituelle Handlungen zu beobachten. Sicher ahnt Goeyvaerts, dass der Übergang zu Massenmedien für die Liturgie ähnliche Konsequenzen haben würde wie der Wandel traditioneller Konzertdarbietungen zu Vorführungen elektronischer Musik, gegen die er von Anfang an große Skepsis heg$\mathrm{te}^{\mathrm{s}}$. Die Vorschläge im ersten Brief an Verheul schließen lückenlos an tragende Ideen seiner seriellen Ästhetik an. Nach wie vor lehnt er die individualistische Kunst, deren Anfang er in der Renaissancezeit situiert, deren wirklich schädliche Auswirkungen bis zur Katastrophe des Zweiten Weltkriegs er in der romantischen und expressionistischen Musik spürt, ab, um ihr ein kollektivistisches Ideal, für das er spätmittel-

48 Brief vom 5. Dezember 1956 an Ambroos Verheul (Archiv K. Goeyvaerts Leuven, II. Briefe, Mappe 5). Sämtliche Zitate aus dieser Korrespondenz sind vom Verfasser aus dem Niederländischen übersetzt.

49 Kennzeichnend dafür der Schlusssatz des erwähnten Briefes an die Familie Stockhausen (22. Dezember 1956): „Und schreibt mir bitte ein bisschen mehr, weil ich die Einsamkeit manchmal schlecht ertrage ..." (Goeyvaerts - Stockhausen. Briefwechsel, hg. von Misch und Delaere [wie Anm. 1], S. 203).

50 Siehe zum Beispiel seine Einschätzung des Konzerts unter dem Titel „Die sieben Stücke“: „Das Konzert in Köln fand am 16. Oktober 1954 statt. Der Name, elektronische Musik' wirkte elektrisierend, und alle, die zur Avantgarde-Elite der Zeit gehören wollten (und davon gab es so manche in Köln), waren anwesend. Es war eine merkwürdige Vorführung: Das Publikum, in die extravagantesten Klamotten gehüllt, folgte mucksmäuschenstill den sehr ausführlichen Erläuterungen Eimerts. Die Musik kam aus zwei riesigen Lautsprecherboxen auf der Bühne, was die Situation noch verrückter machte ... Das kann doch nicht wahr sein! Das ist nicht möglich! Es wurde sofort klar, dass man eine neue Art des Hörens finden musste, eine neue Funktion der Musik ... aber bisher wird elektronische Musik auf diese Art und Weise gehört" (Goeyvaerts: Selbstlose Musik, hg. von Delaere [wie Anm. 4], S. 83-85). 
alterliche Modelle anführt, entgegenzusetzen ${ }^{51}$. Darüber hinaus ist auch angewandte serielle Musik für ihn noch immer elektronische Musik. Daher die benötigte Zusammenarbeit mit Tontechnikern, in diesem Kontext auch Experten der Liturgie, damit persönliche Ausdrucksbedürfnisse zu Gunsten der sakralen Wahrheit zurückgestellt werden können. Goeyvaerts' Ablehnung der Instrumentalmusik beruhte ja auch größtenteils auf dem Ausdruckswillen der Musiker. Sobald auch die Musiker imstande sein würden, zum „selbstlosen Sein“ beizutragen, sah er das Zeitalter von (instrumentaler) serieller Musik angebrochen. Dieses Ideal hatte er bereits in einem Brief an Stockhausen ersehnt:

Der Zustand, den wir vielleicht erreichen können, nach langer Zeit, scheint mir der orientalischen Musik vergleichbar. Der Komponist gestaltet nur ein Schema; das Werk wird von den improvisierenden Musikern weiter gestaltet. Dafür ist aber eine Tradition notwendig. Ein Anfang ist gemacht, wenn Musiker es erreichen, zu spielen ohne dabei ihre Empfindung zum Ausdruck zu bringen ${ }^{52}$.

Stockhausen überträgt diese Idee auf die Komposition elektronischer Musik (während der Arbeit an Studie I):

Es ist unglaublich schön, solche Klänge zu hören, die völlig ausgeglichen, „ruhig“, statisch und dabei nur von strukturellen Proportionen „belichtet“ sind. Regentropfen in der Sonne... [...] Und ich werde in Darmstadt sagen: „Unsere Aussicht: in einem ,Proportionsfeld' improvisierend komponieren" ${ }^{\text {“33. }}$.

In Goeyvaerts' Vorschlag an Verheul ist schließlich auch das Konzept von Musik als Klangstruktur noch immer stark ausgeprägt. „Musik wie sie jetzt geworden ist“ war für Goeyvaerts unabdingbare Voraussetzung für die Produktion liturgischer Musik.

Es bleibt dahingestellt, inwieweit Ambroos Verheul Inhalt und Tragweite dieses Briefs verstanden hat. Jedenfalls war er daran interessiert, Goeyvaerts' Vorstellungen über die Funktion von Musik in neuen Liturgieformen weiter kennenzulernen. Das geht aus seiner Antwort vom 27. Februar 1957 hervor, in der er Goeyvaerts zu einem Studientag am 14. März 1957 mit einer kleinen Gruppe von etwa 25 Seelsorgern, LiturgieExperten, Komponisten und Dichtern, die auf dem Gebiet der neuen Kirchenmusik bereits experimentiert hatten, einlädt. Verheul erwähnt zwar die Enzyklika Musicae sa-

51 Goeyvaerts betonte immer wieder den innerlichen Zusammenhang zwischen serieller Musik und spätmittelalterlicher Kunst, zum Beispiel in „Die Kompositionstechnik im 14. und 15. Jahrhundert in ihrer Beziehung zur Gegenwart“ (Musikalisches Nachtprogramm WDR, 27. Oktober 1957), in: Goeyvaerts: Selbstlose Musik, hg. von Delaere (wie Anm. 4), S. 158-163.

52 Brief vom 30. März 1953, in: Goeyvaerts - Stockhausen. Briefwechsel, hg. von Misch und Delaere (wie Anm. 1), S. 117-118.

53 Brief vom 20. Juli 1953, in: Goeyvaerts - Stockhausen. Briefwechsel, hg. von Misch und Delaere (wie Anm. 1), S. 138 . 
crae disciplina ${ }^{54}$, weist aber dennoch auf die Notwendigkeit von Gemeindegesang und auf das Bedürfnis guter liturgischer Lieder in der Volkssprache hin. „In einigen Kreisen“ sei man aber über die minderwertige Qualität der Volksliturgie besorgt ${ }^{55}$. Hieraus wird deutlich, dass die Zielsetzungen beider Männer von Anfang an verschieden waren. Goeyvaerts belegte den Studientag, formulierte aber am nächsten Tag in einem Brief seine Enttäuschung über die unterbreiteten Vorschläge. Aktive Teilnahme der Gemeinde durch Gesang bringe zweifelsohne einen Gewinn, setze aber eine tiefgreifende Reflektion über das Wesen der Liturgie voraus. Diese habe ihm gefehlt, und deswegen stuft er die Tagungsvorschläge respektive als amorph - wegen des Suchens nach Anhaltspunkten innerhalb des bekannten gregorianischen Idioms - bzw. als vulgär-profan oder total defätistisch ein, da ein Teilnehmer schlechtweg evangelische Kirchenlieder übernommen hatte. Insofern sich aber für die Liturgie eine adäquate Form finden ließe, sei die heutige Musik durchaus imstande diese mitzugestalten:

Dazu sollte man darüber nachdenken, welche Funktion Musik in einem wahren liturgischen Rahmen zu erfüllen hätte. Dieses Problem gleicht jedem Ausdrucksproblem: die Erfindung einer geeigneten Form, die ein geistliches Prinzip ausdruckt. Selbstverständlich sollte man bei dieser Suche auch die praktische Realisierbarkeit in Betracht nehmen ${ }^{56}$.

Mit dieser Formulierung bestätigt Goeyvaerts seine Konzeption serieller Musik als Vertonung eines objektiven, „geistlichen“ Prinzips, nimmt aber auch seine Erfahrungen bei der Realisation seiner elektronischen Werke in Betracht, die ihn für die Idee einer möglichen angewandten, liturgischen Musik erst empfänglich gemacht hatten. Zwischen Verheuls Vorstellung von schlichten Gemeindegesängen in der Volkssprache als musikalische Komponente der Liturgiereform und Goeyvaerts' Ideen zu einer elektronischen Radio- oder Fernsehe-Messe liegt offensichtlich eine unüberbrückbare Kluft. Wie Stockhausen sah Goeyvaerts um 1957 folglich keine Möglichkeit, eine serielle elektronische Messe zu komponieren, die selbst von denjenigen kirchlichen Behörden, die nach einer Liturgiereform strebten, als liturgische Musik akzeptiert und verwendet werden könnte. Eine Messe würde er erst 1968 schreiben, unter völlig anderen Umständen. Goeyvaerts arbeitete damals bereits zehn Jahre als Übersetzer und Texter für die belgische nationale Fluggesellschaft Sabena. Zwar hatte er nach kurzer Unterbrechung, folgend auf eine tiefe persönliche und musikalische Krise im Jahr 1957, sporadisch wieder komponiert, aber vom professionellen Musikleben war er zu dieser Zeit weitgehend abgeschnitten. Dennoch erhielt er vom Flandern Festival den Auftrag, eine Messe zu komponieren. Obwohl Goeyvaerts' Messe formal allen Bedingungen liturgischer Musik entspricht, gehört sie bloß durch diesen Auftrag zur Konzertmusik. Auch wenn sie in

Papst Pius XII hatte diese Enzyklika am 25. Dezember 1955 veröffentlicht. Sie ist gegen romantische Musik in der Liturgie und volkstümliche Messvertonungen gerichtet, und beharrt darauf, dass der gregorianische Choral und die darauf basierten polyfonen Messkompositionen die einzig gültige liturgische Musik seien. Brief von Verheul an Goeyvaerts vom 24. Februar 1957 (Archiv K. Goeyvaerts Leuven, II. Briefe, Mappe 5).

56 Brief vom 15. März 1957 an Verheul (KADOC Leuven, Archiv „Tijdschrift voor Liturgie“, Briefe, Mappe $1.9 / 1,1957)$. 
einem Kirchenraum uraufgeführt wurde und auf liturgischen Texten basiert, beteiligten die Hörer sich damals nicht an einem religiösen, sondern an einem ästhetischen Ritual. Bereits die Idee einer Uraufführung gehört zu diesem Kontext, und darüber hinaus hängt die Wahl eines vierstimmig gemischten Chores und eines doppelten Bläserquintetts ( 2 Oboen, Englisches Horn, 2 Fagotten, 2 Trompeten und 3 Posaunen) mit dem Vorhaben des Festivals zusammen, im selben Konzert auch Igor Strawinsky's Messe (1944-1948), die eine identische Besetzung hat, aufzuführen. Der vollständige Titel dieser Komposition ist aber ein kirchenpolitisches Statement: Mis ter nagedachtenis van Zijne Heiligheid Paus Johannes XXIII. Soweit davon in der katholischen Kirche überhaupt die Rede sein kann - man sei an Stockhausens Dictum erinnert, das Wesen des Katholizismus sei konservativ - gilt Johannes XXIII. (1881-1963) als ein Papst, der versuchte, seine Kirche zu aktualisieren. Er initiierte das Zweite Vatikanische Konzil (1962-1965), das vor allem für die Neugestaltung der Liturgie wegweisend war. Es ermöglichte unter anderem die Liturgie in der Volkssprache neben dem Lateinischen. Daher komponierte Goeyvaerts die fünf Ordinarium-Teile auf einen niederländischen Text. Die Liturgiereform, die Verheul auf lokalem Niveau vorbereitet hatte und auf die Goeyvaerts sich für eine angewandte, kirchenmusikalische serielle Musik Hoffnungen machte, wurde im Vaticanum II gewissermaßen abgesegnet. Nur komponierte Goeyvaerts keine „MitsingMesse" in einem einfachen, populären Idiom, wie sie sich Verheul vorstellte, sondern eine Komposition höheren ästhetischen Anspruchs in der Tonsprache der neuen Musik. Der syllabisch gesetzte Text erhält eine natürliche Deklamation, wird an einigen Stellen (so am Anfang des Kyrie und Credo) geradezu auf einem Ton rezitiert, wird im mehrstimmigen Satz auch des Öfteren im gleichen Rhythmus gesungen, ist also durchaus gut verständlich, aber ansonsten beanspruchen Intonation, Umfang und Setzung der Vokalstimmen und Koordination mit dem Bläserensemble professionelle, mit allen Wassern der neuen Musik gewaschene Sänger statt einer sich musikalisch beteiligenden Glaubensgemeinschaft ${ }^{57}$.

Im Gegensatz zu Stockhausens Gesang der Jünglinge hat die Forschung Goeyvaerts' Messe bis jetzt vernachlässigt, sodass eine kurze analytische Interpretation hier angebracht ist. Der Aufbau eines Messe-Ordinariums weist an sich schon eine gewisse Symmetrie auf. Es liegt nahe, dass der immer konstruktivistisch denkende Goeyvaerts diese Symmetrie als das „Wesen der Liturgie“ betrachtete, das er 1956-1957 noch (vergeblich) von Liturgie-Experten hatte bestätigen lassen wollen. Der Gesamtplan seiner Messe unterstreicht demzufolge erheblich die in der Anordnung der Messe-Teile und in der Textgestaltung vorhandene Symmetrie, wie aus unterstehender Tabelle ersichtlich ist. nis wegen Mangels an Vorbereitung, Kompetenz und Interesse der Musiker katastrophal. Am 9. Oktober 1995 tilgte das Flandern Festival eine alte Schuld, indem es eine Wiederaufführung unter den besten Umständen veranstaltete. Das Konzert durch Collegium Vocale und Champ d'Action unter Leitung von Peter Rundel wurde vom belgischen öffentlichen Fernsehen mitgeschnitten. Die Ironie des Schicksals bewirkte also, dass Goeyvaerts' Messe schlussendlich doch eine „Fernseh-Messe“ wurde, wenn auch nicht unter den liturgischen Bedingungen, die ihm 1956-1957 vorschwebten. 
Tabelle 1: Gesamtplan von Goeyvaerts' Messe.

\begin{tabular}{|l|l|l|l|}
\multicolumn{2}{l}{ Taktanzahl/Tempo } & \multicolumn{1}{l}{ Taktschlaganzahl } & Formale Merkmale \\
\hline Kyrie & $33 / 60-68-60$ & 132 Viertel & dreifacher Aufbau (aba) \\
\hline Gloria & $55 /$ Freudig-bewegt & 328 Achtel [= 164 Viertel] $]$ & $\begin{array}{l}\text { durchkomponiert, viele } \\
\text { Taktwechsel, unregelmäßig }\end{array}$ \\
\hline $\begin{array}{l}\text { Geloofs- } \\
\text { belijdenis }\end{array}$ & $\begin{array}{l}\text { 81/Rasch und rhyth- } \\
\text { misch }\end{array}$ & 302 Viertel & $\begin{array}{l}\text { dreifacher Aufbau } \\
\text { a (durchaus 4/4) } \\
\text { b (durchaus 3/4 aber } \\
\text { sehr unbeständig, } \\
\text { Tonballungen unbestimm- } \\
\text { ter Tonhöhen) } \\
\text { a (durchaus 4/4) }\end{array}$ \\
\hline Sanctus & $\begin{array}{l}\text { metrisch frei / [ohne } \\
\text { Tempo }]\end{array}$ & 74 Dichte-Veränderungen & $\begin{array}{l}\text { a capella, grafisch-räumliche } \\
\text { Zeitandeutung }\end{array}$ \\
\hline Lam Gods & $33 / 92$ & 131 Viertel & dreifacher Aufbau (aab) \\
\hline
\end{tabular}

Nach Umfang und formalen Merkmalen gruppieren sich die Sätze I und V, und II und IV um das zentrale Glaubensbekenntnis, übrigens den einzigen Satz, zu dem einige Skizzen überliefert sind. Die Ecksätze enthalten 33 Takte, möglicherweise einen Verweis auf das Todesjahr Christi. Trotz des konstanten Metrums des Eröffnungssatzes und der vielen Taktwechsel des Schlusssatzes ist die Taktschlaganzahl beider Teile fast identisch. Diese Symmetrie trifft natürlich lediglich auf das Partiturbild zu, nicht auf die, von den verschiedenen Tempi herbeigeführte, unterschiedliche effektive Dauer dieser Sätze.

Die dreifachen Akklamationen der Ecksätze liefern das konstruktive Prinzip, das der ganzen Messe zugrunde liegt. Die drei Klanggruppen Chor - Holzbläser - Blechbläser sind im Tonsatz durchlaufend getrennt. Was in der kirchenmusikalischen Tradition ein Verweis auf Mehrchörigkeit sein könnte, ist hier lediglich Ausprägung des zugrundeliegenden Prinzips. Aus Platzgründen sei hier nur die spezifische Anwendung der Trinität im Eröffnungssatz detailliert erörtert. Wie bekannt, werden Kyrie - Christe - Kyrie je dreimal angerufen, und spiegeln die Kyrie-Teile sich symmetrisch um den Mittelteil. Für Goeyvaerts wäre eine bloße Wiederholung des Kyrie nach dem Christe aber zu schwach, zu wenig mit den "geistlichen Grundlagen" sowohl des Serialismus wie der Liturgie übereinstimmend. Deswegen gestaltet er das Kyrie II als krebsläufige Wiederholung des Kyrie I, wobei nicht nur die Tonhöhen (inklusive Oktavlagen), sondern auch die Dauern, die Dynamik und die Artikulation in den Rücklaufprozess miteinbezogen sind. Nichts hebt den Prozesscharakter von Musik emphatischer auf als ein Krebsgang. Noch verstärkt durch das punktuelle Klangbild, in dem isolierte Töne oder kurze Tongruppen stark kontrastierende Parameterwerte und Oktavlagen haben, kreiert Goeyvaerts eine Konstellation, in der "tote" Klanggegenstände, also Tonpunkte mit gleichbleibenden Klangeigenschaften, symmetrisch im musikalischen Raum verteilt sind. Umkehrung der Zeit bedeutet Zeitlosigkeit, Aufhebung der Zeit, Stasis, keine Entwicklung sondern Rückkehr zum Anfang, oder - in Goeyvaerts' theologischem Konzept - Darstellung der Ewigkeit Gottes. 
Über die krebsgängige Anordnung der beiden Kyrie-Teile hinaus weist auch die innere Gestaltung des Kyrie I auf die symmetrische aba-Form, die im Textaufbau vorhanden ist (siehe Notenbeispiel 1).

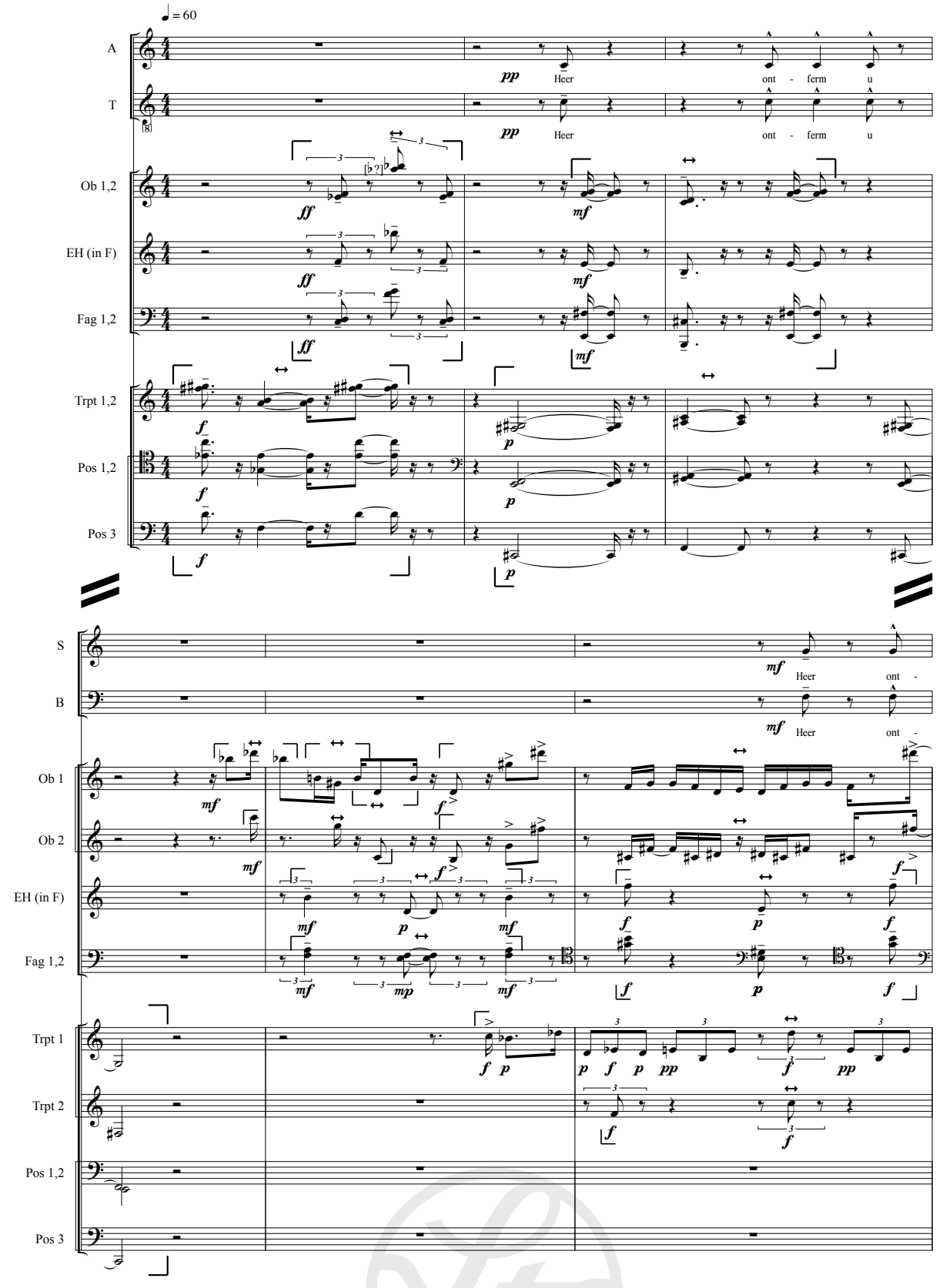




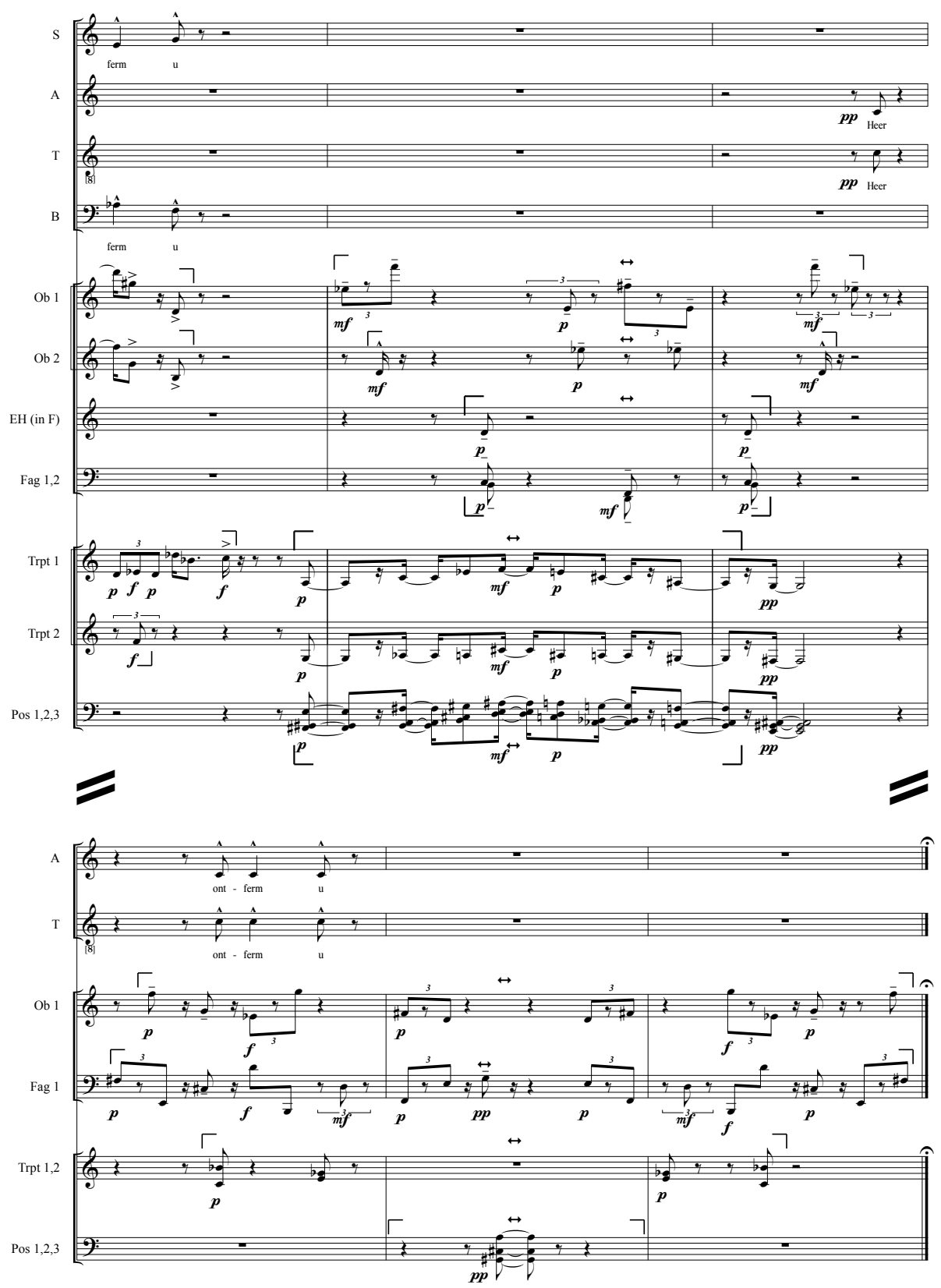

Notenbeispiel 1 (diese und vorherige Seite): Goeyvaerts, Messe. Kyrie I, T. 1-12. 
Die dreifache Anrufung („Heer ontferm U“) ermöglicht eine sowohl zeitliche wie auch räumliche symmetrische Setzung im Chorsatz. In der ersten und letzten Anrufung rezitieren die mittleren Alt- und Tenor-Stimmen das c', in der zweiten Anrufung umsingen die äußeren Sopran- und Bassstimmen in Gegenbewegung symmetrisch diesen Mittelpunkt. Der musikalische Raum eröffnet und schließt sich also um einen Nullpunkt herum. Auch die Instrumente prägen Trinität und Symmetrie in verschiedenen Dimensionen aus. Der Instrumentalsatz, in dem Holz- und Blechbläser durchaus klar getrennt sind, ist charakterisiert durch die Abfolge Blöcke (T. 1-4), kontrapunktischer Satz (T. 5-7; in den tiefen Holzbläsern kombiniert mit Blöcken) und Punkte (T. 8-12; in T. 8-9 und 11 in den Blechbläsern kombiniert mit Blöcken). Die Klangmaterie wird also erst kompakt vorgestellt, erfährt dann ein zeitlich-räumliches Zerfließen, um sich schließlich in Einzelpunkte aufzulösen. Selbstverständlich kehrt diese Aufeinanderfolge sich wegen des Krebsgangs im Kyrie II um. Die Musik geht nirgendwo hin und kehrt zu sich selbst zurück, sie ist lediglich eine statische, proportionale Ausweitung und Zusammenschrumpfung eines Klangmaterials in Raum und Zeit.

Es lohnt sich auch, das Klangmaterial auf der Mikro-Ebene zu untersuchen. Das Muster der Akkordblöcke in T. 1-4 entspricht dem Textaufbau des Kyrie-Satzes (aba). Es klingen jedes Mal drei Akkorde: ein Akkord, dessen Transposition und die Wiederholung des ersten Akkords (aba). In den Blechbläsern erfolgt die Transposition in T. 1 nach unten (Muster hoch-tief-hoch), und bei der nächsten Dreiergruppe in T. 2-4 nach oben (tief-hoch-tief; in Augmentation). In den Holzbläsern finden sich dieselben Merkmale in umgekehrter Richtung, wobei die Tief- und Hoch-Transpositionen im Quintintervall darüber hinaus die symmetrische Spiegelung der Vokalstimmen um den Achsenton c' präfigurieren. Die Akkordblöcke in den tiefen Holzbläsern T. 5-6 stehen nicht länger in einem Transpositionsverhältnis, weisen aber immer noch das aba-Muster auf. Ihre dynamische und rhythmische Anlage stimmt mit dem Hauptmerkmal des kontrapunktischen Abschnitts T. 5-7 überein. Tatsächlich spielen Oboen und Trompeten eine Aufeinanderfolge und Überlagerung symmetrischer aber verschieden dimensionierter Zellen (in Notenbeispiel 1 durch Klammer und Achsenpfeile angedeutet). Lediglich die kürzeren, dreitönigen Zellen zeigen noch das aba-Muster (T. 5: Oboe 1, gewissermaßen auch Oboe 2; wie oben gesagt T. 5-6: Englisches Horn, Fagotte; T. 6-7: Trompete 2), aber ansonsten handelt es sich lediglich um rückläufige symmetrische Zellen unterschiedlichster Größe. Sämtliche Klangdimensionen sind dabei berücksichtigt. Als Beispiel davon sei hier auf Trompete 1 in T. 5-7 verwiesen. Wie bei den anderen Zellen bezieht der Rücklauf sich hier auf Tonhöhe, Dauer, Artikulation und Dynamik, wird aber durch die ständige Abwechslung der dynamischen Werte besonders hervorgehoben. Auch der letzte Abschnitt („Punkte“) in T. 8-12 enthält lediglich verschieden proportionierte, krebsgängige symmetrische Zellen. Nur die Akkordblöcke in den Blechbläsern T. 8-9 weichen leicht davon ab, indem ein Zusatzakkord und die nicht völlig einwandfreie Spiegelung der Tonhöhen die symmetrische Anordnung dieser Akkorde in den Bereichen Dauer und Dynamik ausnahmeweise schwächen.

Die Allgegenwärtigkeit der Spiegelsymmetrie ist aus mindestens drei Gesichtspunkten einleuchtend. Obwohl Goeyvaerts 1958 im Einführungsreferat zum Münchner Fes- 
tival über serielle Musik diese als ein „zum Tode führendes Schiff“ bezeichnete ${ }^{58}$, sind Grundprinzipien des seriellen Denkens in seinen späteren Kompositionen, wie auch in seiner Messe, noch immer gültig. Vor allem das Konzept einer statischen Musik ist nach wie vor ausgeprägt: „das musikalische Werk als Projektion in Zeit und Raum einer Grundidee, welche die Struktur erzeugt" ${ }^{\text {59 }}$. Das Kyrie zeichnet sich in dieser Hinsicht besonders aus, da es die im Text vorgegebene Idee einer ternären Spiegelsymmetrie in allen Dimensionen, von der Großform über die Instrumentation und den Tonsatz bis zu den kleinsten Zellen, sowohl vertikal wie horizontal, also in Raum und Zeit, zum Ausdruck bringt. Zweitens haften Symmetrie und Trinität Symbolwerte von Perfektion und Ausgewogenheit an, die für Goeyvaerts' ästhetisch-theologisches Denken bedeutend sind. Schließlich sollten noch die Vorteile innensymmetrischer Strukturen für die Bildung einer Krebsform berücksichtigt werden. Kompositionstechnisch ist die Retrograde eine Herausforderung, die durch die Verwendung symmetrischer Zellen in Zeit und Raum - wodurch „recto“ und „verso “ auf dieser Ebene identisch sind - etwas erleichtert wird. Dieses sonst schwer wahrnehmbare konstruktivistische Prinzip wird dadurch sogar einigermaßen hörbar ${ }^{60}$.

\section{Fazit}

Die vom Katholizismus geprägten Biografien Stockhausens und Goeyvaerts' lassen vermuten, dass sie die Komposition einer Messe nicht nur aus „Pflicht“, sondern auch aus „Neigung“ hätten angehen können. Nicht ihr Glaube, sondern dessen spezifische Ausprägung in seriellen Werken hielt sie aber vorerst von diesem Vorhaben ab. Zwar formulierte Bernhard Uske den Verband zwischen serieller Musik und der katholischen Liturgie eindrücklich, wenn er schreibt:

Eine Liturgie, die vor ihrer Verstümmelung durch die Liturgie-Reform mit ihrer minutiösen Regelung von Gesten, Farben, Formen, Zeichen und Gesängen selber Züge des Seriellen hatte und - verstanden als Inkarnation des Logos, als Realpräsenz der göttlichen Ewigkeit in der Zeit - die Makro-Performance des Heiligen war $^{61}$.

Für Goeyvaerts und Stockhausen waren die „geistlichen Grundlagen“ des Serialismus in der Pionierzeit aber zu strikt, um eine verwirrende und vor allem unkontrollierbare Vielfalt an Gesten, Farben, Formen, Zeichen und Gesängen als vertretbare Darstellung ren. Ein berüchtigtes Beispiel liefert Arnold Schönbergs „Der Mondfleck“ aus Pierrot lunaire Op. 21 (1912).
Siehe dazu Mark Delaere, Funktionelle Atonalität. Analytische Strategien für die frei-atonale Musik der Wiener Schule (Veröffentlichungen zur Musikforschung 14), Wilhelmshaven 1993, S. 85-97.

61 Bernhard Uske, „Klang statt Kirche: Warum ist die neue Musik so religiös?“, in: NZfM 163, 2002, H. 5, S. $13-14$. 
des Göttlichen zu akzeptieren. Sobald aber die Unmöglichkeit einer hörbaren Durchrationalisierung anerkannt wurde - und dies war bei Stockhausen viel früher der Fall als bei Goeyvaerts - stand der Komposition einer Messe prinzipiell Nichts mehr im Wege. Nur nach Auflockerung des musico-theologischen Reinheitsgebots also waren die Voraussetzungen für eine angewandte serielle Musik geschaffen. Aus der Perspektive beider Komponisten eignete sich dafür nichts besser als die Produktion liturgischer Kirchenmusik. Stockhausen fasste 1954-1955 als Erster den Plan, eine Messe für Stimmen und elektronische Klänge zu komponieren. Bald wurde er mit der Unmöglichkeit einer liturgischen Anwendung dieser Musik konfrontiert, obwohl er sich darüber nicht direkt mit den liturgischen Behörden auseinandergesetzt zu haben scheint. Da eine Messe außerhalb des liturgischen Kontexts ihm sinnwidrig erschien, wandelte er sein ursprüngliches Vorhaben in die Tonbandkomposition Gesang der Jünglinge um. Durch die Verwendung des „Benedicite“ als Textvorlage dieses Werks, behielt er zwar einen Bezug zur Liturgie bei. Dieser Bezug ist aber eher gedanklich denn konkret, da Stockhausen sich damals kaum die Wiedergabe von Gesang der Jünglinge für liturgische Zwecke hätte vorstellen können. Es gab aber nicht nur die Vorbehalte gegen die liturgische Verwendung einer elektronischen Messe, darüber hinaus war das „Benedicite“ eher Zusatz denn fester Bestandteil der Messliturgie, und eine Aufführung in deutscher Sprache wäre 1955-1956, in der prä-konziliären Ära also, in einem liturgischen Rahmen überhaupt kaum möglich gewesen. Auch wenn er auf einem sakralen Text basiert, ist der Gesang der Jünglinge emphatisch Konzertmusik.

Stockhausens Vorsatz, eine elektronische Messe zu komponieren, stieß bei Goeyvaerts nicht auf taube Ohren. Im Gegensatz zu seinem Freund trat er 1956-1957 in Kontakt mit Experten der Liturgie, um ihnen hinsichtlich seiner Pläne den Puls zu fühlen. Von der Liturgiereform, die während der 1950er Jahre vorbereitet und später im Vaticanum II konkretisiert wurde, erhoffte er sich ein radikales Umdenken des sakralen Rituals in Richtung einer zeitgemäßen kirchenmusikalischen Praxis. Die entmenschlichte, rational kontrollierte elektronische Musik schien ihm das ideale Medium für eine neugestaltete Liturgie, die sich (auch) als Radio- oder Fernsehe-Messe zelebrieren ließ. Das setzte aber die Zusammenarbeit von Komponisten, Tontechnikern und Liturgie-Experten voraus. Goeyvaerts' Hoffnung erwies sich bald als naiv, sodass er seine Ideen zu einer seriellen, elektronischen Messe damals nicht weiter entwickelte. Etwas mehr als zehn Jahre später komponierte er trotzdem eine Mis ter nagedachtenis van Zijne Heiligheid Paus Johannes XXIII, die durch die Verwendung der Volkssprache äußerliche Züge der vom zweiten Vatikanischen Konzil eingeführte Liturgiereform aufweist, in deren strenger, hieratischer Gestaltung aber keineswegs die erstrebte Volksnähe verwirklicht ist. Stilistisch ist Goeyvaerts' Messe insgesamt vielleicht eher hybrid, aber die Gestaltung des Kyrie zeigt, dass eine serielle Kompositionstechnik und Ästhetik sich durchaus auch in angewandter Form, eben als funktionale liturgische Musik, realisieren ließ.

MARK DELAERE

University of Leuven

mark.delaere@arts.kuleuven.be 